\title{
An experimental assessment of the saturated transverse permeability of Non-Crimped New Concept (NC2) multiaxial fabrics
}

\author{
O. Elbouazzaoui ${ }^{\dagger}$, S. Drapier ${ }^{\dagger}$, and P.Henrat ${ }^{\ddagger}$ \\ ${ }^{\dagger}$ Mechanical and Materials Engineering Department - SMS Division \\ École Nationale Supérieure des Mines de Saint-Étienne \\ 42023 Saint-Étienne Cedex 02, France \\ $\ddagger$ Hexcel Reinforcements \\ ZI Les nappes - 38630 Les Avenières, France
}

\begin{abstract}
Over the past few years were developed some manufacturing processes involving resin impregnation in dry preforms prior to cure, and more specifically resin infusion across the fabric thickness in the so-called Resin Infusion Processes. Therefore, in these growingly used processes the transverse permeability of the fabrics will control both manufacturing cycles and dimensions, and consequently the mechanical properties of the final composites. A big effort has been realized to obtain reliable experimental techniques for measuring the transverse permeability. Since the differentiation between saturated and effective permeabilities is still discussed, and the flow front is hardly defined in such small dimensions (order of $1 \mathrm{~mm}$ ), our measurements on some so-called Non-Crimped New Concept $(\mathrm{NC} 2)$ are carried out for saturated flow, i.e. the fiber network is completely impregnated from the onset of the experiment. In our case [1], these measures rely on the measurements of the pressure drop induced by the flow of a controlled fluid across the fabrics, easily converted into transverse permeability through the Darcy's law. One of the main results of these measurements is that the low $\mathrm{NC} 2$ transverse permeability depends on the face of the fabric receiving the fluid. This original behaviour may, in turn, be translated into manufacturing guidelines to achieve optimal processing configuration. An attempt to relate this differential to the stitching hole surface is proposed which turns to explain minor phenomena. On the contrary, the 3D shape of the stitching hole reconstruction from polishings could yield some proper explanations.
\end{abstract}

Keywords: Experimental assessment, Transverse permeability, NC2 (Non-Crimped New Concept), Saturated regime, Darcy

\footnotetext{
${ }^{1}$ Author to whom correspondence should be addressed: Tel: +33-04-77-42-00-79. Fax: +33-04-7702-49. E-mail address: drapier@emse.fr.
} 


\section{Introduction}

The so-called Liquid Composite Moulding (LCM) technologies, such as resin transfer moulding (RTM), structural resin injection moulding (SRIM), SCRIMP, etc..., are more and more widely used for producing fibre reinforced polymer composites. These techniques offer low cost, low solvent emission, along with high and reproducible quality and versatility. In these processes, the dry preform is first placed in a mould, then the liquid resin is injected under constant pressure or velocity, and eventually the resulting compound undergoes a cure cycle. A unique feature of those processing techniques is that the liquid but viscous resin has to flow a long distance to impregnate the dry fibres. This may cause the formation of voids or resin distribution heterogeneities which translate into dimension inaccuracies or mechanical properties alterations.

An alternative to these problems of long dimensions to be injected, is to impregnate the preforms over their wide in-plane surface. Among these processes, Resin Infusion Processes (RFI, RIFT, LRI, ...) [2], [3], [4], [5] have been identified as cost-effective methods for producing composite parts. One great advantage of this type of technique is to permit the elaboration of complex-shaped components [6]. But in counterpart, some difficulties arise for controlling the dimensional accuracy, even if for simple parts metal toolings can be placed on either side of the stacking to get well-finished surfaces (Figure 1). In these processes, resin layers (solid or liquid) are stacked with the preforms in a mould (Figure 1-a). The whole stacking is then vacuum bagged and usually placed in an autoclave to undergo a temperature-pressure cycle (Figure 1-b). It is clear that in this process, the filling time, the resin heterogeneity distributions, the fibre volume fraction, and therefore the final thicknesses (Figure 1-c), will depend mainly on the resistance of the preform against the resin flow, i.e will depend on the permeability properties across the thickness. However, this permeability of the fabrics across the thickness is not as studied as the in-plane permeabilities. One of the specificities of this parameter is that it must be assessed, unlike in-plane permeabilities, on very short dimensions. Meanwhile accurate permeability data are essential for the process simulations.

[Figure 1 about here]

The present paper is dedicated to the measurement of the transverse permeability in long-fibre composites, more precisely in new multiaxial stitched semi-products developed by Hexcel Reinforcements called Non-Crimped New Concept $(N C 2)[1]$. In this 
study we present the measurement of transverse permeability of various $N C 2$ semiproducts, chosen according to some industrials applications they are intended for : Automotive, Aeronautic, and Wind energy. Regarding the results obtained from the measurements, and especially the dependance of the permeability on the face receiving the fluid, the morphology of the stitching holes is also studied.

\section{Permeability characterization}

The permeability of a medium represents its resistance against a fluid flow. Since composites can be seen as multi-scale porous media, this permeability can be defined at various scales.

\subsection{Scale of observation and associated permeability descrip- tion}

At the component scale CARMAN \& KOzENY [7] [8] have proposed a model through which the permeability of fibrous composites is related to the porosity defined as the ratio of open-pores over the entire volume considered. This model is widely used $([9],[10],[11], \ldots)$ but it is important to point out that, although it never appears in the various studies relying on this model, the KOzENY's constant can vary, depending whether the matrix (solid phase) is solid or fibrous [7]. All this is difficult to figure out, and this model is rather used empirically and for fibre networks showing a homogeneous fibre distribution.

Moreover, it must be noticed that the porosity on its own requires a considerable amount of work, usually feeded into statistical studies of the pore distribution [12] [13] or conceptual models [10] [14]. For industrial purposes, and in the case of our stitched multiaxial materials, such approaches are prohibitive. A macroscopical approach is preferably used which describes the global fabrics permeability, with the risk of neglecting any local effect that may cause permeability variations, but the origin of which will appear indirectly.

The most popular macroscopical approach, based on experimental observations of unidirectional flows, has been proposed by DARCY [15] to describe the permeability of 
sand columns to a water flow, it writes :

$$
Q=k \frac{A}{\eta} \frac{\Delta \hat{P}}{\Delta L}
$$

where $\mathrm{Q}$ is the fluid flux $\left(m^{3} s^{-1}\right), \Delta \hat{P}$ is the pressure (hydraulic head) differential in the flow $(P a), \Delta L$ is the flow length $(m), \eta$ is the fluid dynamical viscosity $(P a s), A$ is the flow cross-sectional area $\left(m^{2}\right)$, and $k$ is the porous medium permeability $\left(m^{2}\right.$ or DARCY). This was later generalized for 3D flows where the second order permeability tensor is described by 9 coefficients, as it is supposed to be symmetrical. A usual further restriction is to consider composite semi-products as orthotropic regarding their aspect exhibiting a preferential plane in which the flow can propagate. Then as a first approximation, only three independent constants define the permeability tensor in its principal directions : two in-plane (longitudinal) permeabilities and one (throughthickness) transverse permeability.

Obviously, the Darcy's law which is a particular case of the Brinkman's law for laminar flows, has some limits that must be verified when using it (see [7], [8] for details). More especially for composite impregnation the following points are of importance. A newtonian and incompressible fluid is considered [16], this is justified if the infusion (or more generally the impregnation) takes place before the resin gelification. The porous medium is infinitely rigid, this can be questioned for fabrics where compaction may take place during the flow [17]. The flow must be laminar, this is usually verified for infusion processes. The porous medium is fully saturated in fluid, which is obviously violated during the filling where a partially saturated zone exists in the vicinity of the flow front [12]. The pore size is small with respect to the porous medium dimensions. Eventually, permeabilities are intrinsic to the medium, independant of the fluid nature, and the effects of diffusion and capillarity are neglected. These restrictions may not be satisfied in transient regime.

It appears that, depending on the regime of saturation, the fluid flow may differ considerably. Therefore, it is worth reporting the differences in the characterization of permeability in these two regimes, and even first to have a look at the literature.

\section{$2.2 \quad$ Regime of measurement}

Let us first recall that the permeability describes a flow in a porous medium. As demonstrated both theoretically and experimentally on ideal arrangements by SAID \& Phelan [18], Phelan \& Wise [19] or Sadiq et al. [20] and Parnas et al. [21], this 
flow results from the balance between the viscous forces of the fluid and the capillary forces between the fluid and the skeleton. Moreover, it is clearly demonstrated [21] [22] [10] that this flow occurs at several scales in real composites. Even though these studies focus on some materials which may be different from the composites industrially used, clearly the permeability is a function of the fluid viscosity and velocity, of the surface tension, and especially of the saturation of the medium which produces some changes in the surface tension [23].

As far as the authors know very few studies make this distinction of saturated/unsaturated regimes in measuring the permeability on the same material of industrial interest. One can report the plane permeabilities measured by MA \& SHISHoo [24] who show a ratio $\frac{k_{u n s a t}}{k_{\text {sat }}}$ around 0.7 for some mats, and 0.4 for some orientated materials. However, LUNDSTRÖM et al. [25] gathering some plane permeability measurements obtained through three different methods, two in saturated regimes and one in transient regime, reports that no significant difference can be observed. In the same way, HAMMOND \& Loos observe the same permeabilities in testing glass weaves and satin carbon fabrics with several fluids [26]. On the contrary, BALATTA et al. [27] and LuCE et al. [28] show that the transverse permeability measured for glass mats are lower in saturated regime than in transient regime, with a ratio $\frac{k_{\text {unsat }}}{k_{\text {sat }}}$ of 4 . This tendency is confirmed by BinÉtruy \& PABIOT [22] who verify experimentally and by a model, that the ratio $\frac{k_{\text {unsat }}}{k_{\text {sat }}}$ is of the order of 1.5 to 2 for some stackings of woven and mats glass fabrics, with some fibre volume fraction ranging between 40 to $50 \%$. Finally, the distinctions between saturated and unsaturated regimes are usually made for in-plane permeabilities only, and the question is still open.

\subsection{Transverse permeability measurements}

Following the previous remarks upon the flow regime, there are two common types of methods for measuring the permeability of fabrics and preforms, both based upon the DARCY 's law (Equation 1). These are namely the steady state and advancing front measurements.

Transverse permeabilities are usually characterized for steady-state regimes [29] [17] [1], based on a unidirectional flow. It can be stated that the transient permeability will be more appropriated for describing the real injection processes. But this transient permeability relies usually on optical observations of the flow front [10], quite difficult to achieve on such small distances (of the order of the $m m$ for our $N C 2$ ) and very 
seldom purely unidirectional regarding the capillary effects that may drive the flow [23]. To overcome this problem, 3D front tracking are used and transverse permeability is back-calculated from mass conservation expressions combined with Darcy's law [17] [30] [12] [31]. This implies to know the in-plane permeabilities and yields also complex experimental and numerical problems.

For the sake of simplicity, and since the materials used here are not known, a specific apparatus was developed for the measurement of the saturated transverse permeability using a unidirectional flow through-the-thickness [1]. Relying on the DARCY 's law for 1D flows (Equation 1), the permeability is deduced knowing the fluid velocity and the pressure differential, the elevation change being neglected due to the reduced flow length. The fluid velocity is derived directly from the inlet rate flow, and the pressure differential is deduced from the discrete measurements of the pressure along the flow (Figure 2). Finally the permeability is given by :

$$
k=\frac{A}{\eta Q} \frac{h}{P_{\text {in }}-P_{\text {out }}}
$$

where $h$ is the specimen thickness, and $P_{\text {in }}$ and $P_{\text {out }}$ are respectively the inlet and outlet pressures.

[Figure 2 about here]

\section{$3 \quad$ Experimental details}

The experimental data presented here are mostly detailed in [1], apart from some improvements brought to the testing device. The present section will focus mainly on the validation of the experimental apparatus, especially in the case of our very little permeable $N C 2$ fabrics.

\subsection{Sample preparation and testing device}

Mainly, the transverse permeability measurements will permit to optimise the Resin Infusion Processes used in industry with the Non-Crimped New Concept (NC2) multiaxial semi-products. Three families of $N C 2$ were selected according to the different industrial applications aimed at by Hexcel Reinforcements which supplies these reinforcements: Automotive, Aeronautic, and Wind energy. The fabric faces can be seen in Figures 3. Complete data about these materials are given in Table 1. 
[Table 1 about here]

[Figure 3 about here]

As explained previously, the principle on which this device developed and detailed in [1] relies (Figure 4) is quite simple (Figure 2). It permits to deduce the apparent transverse permeability by measuring a fluid pressure drop induced by the flowing across a fabric. The whole technological realization comprises a micro-gear pump, controlled by a PC, for the fluid injection into the lower chamber of the apparatus. Pressure data are measured in the upper and lower chambers using some probes $\left(\right.$ ASHCROFT $^{\circledR}$ type KXF) connected to a National Instruments data acquisition system controlled by LabVIEW ${ }^{\circledR}$ software. The fixture test section was designed to characterize $100 \mathrm{~mm}$ diameter fabric preform samples. The upper chamber and lower chamber contain porous surfaces (aluminium honeycomb) allowing the fluid flow through the thickness of the specimens while maintaining them. Compared to the fixture as presented in [1], for a better oil tightness two intermediate honeycomb plates equipped with a toric joint were added which form an intermediate chamber containing the fabric tested. These complementary plates ensure the stability of the measure even for heavy materials as those considered here, provided clamping can sustain the pressure induced by the fluid flow. The stability of the measures was verified by controlling that both pressures in the fluid would not change, even for long periods of injection.

[Figure 4 about here]

Thanks to these extra plates a perfect reproducibility of the measures is obtained as it has been verified in testing repeatedly metal grids, woven fabrics, and $N C 2$ fabrics. But the introduction of these parts has some drawbacks. First, the cutting that was initially integrated to the apparatus to avoid some discrepancies due to the handling of the fabrics can no longer take place in-situ. But the results presented below demonstrate that a good repeatability can be conserved. Second, the fabric thickness, and therefore the fibre volume fraction, is no longer prescribed, but it is estimated through some combinations of measurements of the fabric compaction response and of a mechanical model representing the non-linear stiffnesses of the compacted stacking (fabric + joints + plates $)$. 


\subsection{Employed protocol}

The objective of this protocol is to follow the change in the hydraulic resistance of the saturated multiaxial $N C 2$ as a function of different flow rates. Since we have no information about the resin infusion velocity occurring in the industrial process, the flow rates considered here range from 0 up to occurrences of leakage in the apparatus.

In comparison with the protocol used in the initial version of the setup [1], here the circular specimen is first placed in the extra honeycomb tightness plates, and this stacking is then placed in the testing chamber of the setup. Then the measurement protocol can start which is divided in three steps as represented in Figure 5 where are plotted the normalized flow rate, injection pressure, and outlet pressure as functions of time. In the first times of the cycle the hydraulic set-up is filled up with a low flow rate $(0.1 \mathrm{l} / \mathrm{min})$ until the sample completely saturates (part I in Figure 5). Then the fluid injection is stopped (part II in Figure 5). In the third step a series of measures is carried out by increasing step by step the flow rate and recording the pressures on both sides of the sample.

[Figure 5 about here]

\subsection{The test fluid}

The test fluid used is a commercial Neutral oil (ISO 150), the behaviour of which is newtonian [29]. This fluid was chosen for its viscosity that is of the order of the viscosity of standard thermosetting resins in processing conditions $(\sim 0.06 \mathrm{~Pa} s)$. Moreover this fluid is chemically non-aggressive towards the samples and set-up, and presents a low cost and ease of cleaning and disposing, unlike the resins that are actually used in a production cycle.

The fluid viscosity was measured in the temperature range $15-25^{\circ} \mathrm{C}$, and can be fitted by the following fourth degree polynomial:

$$
\eta(T)\left[10^{-3} \mathrm{Pas}\right]=9.6955 E^{-6} T^{4}-1.8403 E^{-2} T^{3}+0.88167 T^{2}-25.782 T+3.4761
$$

where $\mathrm{T}$ is the room temperature in ${ }^{\circ} \mathrm{C}$. In our experimental conditions, the oil temperature was checked with an in-situ thermocouple, and appeared to be constant. At this room temperature of $25^{\circ} \mathrm{C}$ the viscosity is $0.0615 \mathrm{~Pa} \mathrm{~s}$. 


\subsection{Validation of the $N C 2$ transverse permeability measure- ments}

As indicated previously (Equation 2), the transverse permeability is identified from the measured flow rates and corrected pressures. Indeed, the pressure drop inherent to the device has to be removed from the pressure sensor measurements. This pressure drop, that is mainly due to the honeycombs, was checked to be independent on the fluid velocity and is worth 0.014 bars, value of the order of the pressure sensors precision.

In order to assess the repeatability and to verify the applicability of the DARCY 's approach, we plot the results as some resistance curves, representing $\frac{\Delta P}{\eta h}$ versus the superficial velocity $U\left(=\frac{Q}{A}\right)$, such as presented in Figure 6 for instance for some Automotive $N C 2$ fabrics. Then the apparent permeability, noted henceforth $k$, is the inverse of the slope of these curves. One will notice that for 0 fluid velocity, the pressure drop is not zero. It can be suggested that for these low velocities, capillary effects may prevail in the flow control, yielding a non-linear response which turns out to be quite tricky to grasp in saturated regime.

[Figure 6 about here]

From these curves, one can estimate the global discrepancy of the measurements for the various specimens tested, but also the linearity of the individual responses which ensures that the DARCY 's law holds. Both can be assessed using an error estimation, noted $E(U)$, representing the difference between the experimental points for any fluid injection rate and a linear regression fitting these points in the sense of the least squares.

Applying these error estimates (or distance to the linearity) to the experimental measurements reported in Figure 6 yields the following results of Table 2. Clearly, for the three specimens tested for this material, the individual response is definitely linear, the mean error $\bar{E}(U)$ being $4 \%$ at the maximum. Globally, as could also be expected from the graphic representation, the repeatability is excellent with a mean error (for a global linear regression) lower than $4 \%$. It can be concluded that both the use of the DARCY 's law is legitimate and the repeatability is verified. Moreover, it must be pointed out that these discrepancies on the resistance curves will not have the same implications on the permeability that corresponds to the slope of the linear approximations.

[Table 2 about here] 
On this particular example, the repeatability is well verified, and we shall verify this for the three $N C 2$ types tested. As it will be seen below, the variation in the measurements carried out in the present study will never exceed $15 \%$ for the most sensitive $N C 2$. This has to be compared with the results from LunDSTRÖM et al.[32] for whom the repeatability is $15 \%$, but for some in-plane flows assessed over $300 \mathrm{~mm}$ long distances.

\section{Transverse permeability measurements of the $\mathrm{NC} 2$ fabrics}

Prior to the permeability measurements, the fabric thickness was estimated for a clamping force of 3,234 N, corresponding to the largest air pressure that can be applied to the clamping actuator. The estimated thicknesses are reported in Table 3 where appear the initial fabric thickness, the compacted thickness, and the corresponding fibre volume fraction. The consistency of the thicknesses estimation was validated through multiple measurements, especially displacement-force response of the dry fabrics, and direct and indirect measurements of the initial thicknesses. From this Table 3, one verifies that the fibre volume fractions, as well as the equivalent compaction pressure that would lead to such fibre volume fractions (deduced from compaction experiments), are consistent with the industrial process conditions (industrial compaction pressure around 3 to 5 bars).

[Table 3 about here]

Once the thickness of the fabrics is known, the resistance curves can be plotted for every $N C 2$ type selected for this study.

\subsection{Resistance curves and permeability differential phenomenon}

For every measure of permeability, six different fabrics were tested for each family of $N C 2$ fabrics. The results are reported in the following paragraphs.

\subsubsection{Resistance curves for the Automotive $\mathrm{NC} 2$}

In Figure 7 are plotted the resistance curves for the Automotive applications. One can verify the very good repeatability and linearity for this biaxial NC2. But above all, 
these results reveal that the pressure drop induced by the flow across the $N C 2$ fabrics, and therefore the permeability itself, depends on the face receiving the fluid. This original and surprising differential of permeability has never been reported in the literature, even for close meso-structures such as the $N C F$ multiaxial reinforcements for instance. This characteristic behaviour observed for every measurement made on the $N C 2$ led us to distinguish the $N C 2$ faces, noted $O O$ for the face where the stitching loops lay (see Figure 3-b), and ZZ for the face where the stitching can be in zig-zag or straight (see Figure 3-a).

[Figure 7 about here]

Obviously, this differential of permeability was studied in depth, it appears to be reproducible and reversible. As can be seen in Figure 8, the linearity of the resistance curves is preserved when the the injection rates are increased and then decreased, the mean distance to the linearity being $7.5 \%$ for the $Z Z$ face and $5.9 \%$ for the $O O$ face for this particular case. Therefore, this differential of permeability cannot be an artefact, but is an original characteristic of the $N C 2$ multiaxial reinforcements.

[Figure 8 about here]

The treatment of the results for these Automotive $N C 2$ is reported in Table 4 . The very good reproducibility and linearity of these $N C 2$ clearly appear. Individually, the distance to the linearity (mean error $\bar{E}(U)$ ) varies between 2.9 and $7.6 \%$ for $Z Z$ faces and 1.4 to $4.1 \%$ for $O O$ faces. This error is perfectly acceptable and demonstrates that the flow regime is of DARCY's type. Moreover, the repeatability is verified since the distance to the global linear fit reaches only $6.7 \%$ for the $Z Z$ faces and $3.9 \%$ for the $O O$ faces.

[Table 4 about here]

Finally, these results for the Automotive $N C 2$ show that the resistance to the flow is a linear function of the fluid velocity. Moreover, this resistance depends strongly on the face receiving the fluid, this effect being reversible and reproducible. The whole results are quite homogeneous, certainly due to the low weight of these biaxial $N C 2$, but also to the low sensitivity to the handling resulting from the good support of the $0^{\circ}$ fibres provided by the zig-zag stitchings (see Figure $3-a$ ). 


\subsubsection{Resistance curves for the Wind Energy $\mathrm{NC} 2$}

For these glass fibre $N C 2$, regarding the very low support to the fibres on the $0^{\circ}$ face (Figure 3-b) and the high weight of the UD fabrics, the results are strongly related to the quality of the specimen cutting (Figure 9 face $O O$ ). Again, in these permeability measurements the dependance on the face injected is verified (Figure 9), with this time, unlike for Automotive $N C 2$, the $O O$ face inducing higher pressure drops than the $Z Z$ face. Also, when compared to those previous $N C 2$ (Figure 7), at fixed fluid velocity Wind Energy $N C 2$ induce pressure drops that are twice as large for the least permeable face $(O O)$, and comparable for the most permeable face $(Z Z)$.

[Figure 9 about here]

Here, the distance to the linearity is larger than for Automotive $N C 2$, at least for the $O O$ face, even if the individual error remains acceptable (Table 5), comprised between 0.8 and $4.1 \%$ for the $Z Z$ faces and 3 to $12.5 \%$ for the $O O$ faces. On the contrary, the distance to the global linearity becomes relevant, at least for the $O O$ face $(30 \%)$ while remaining acceptable for the $Z Z$ face (12\%). These discrepancies can be directly related to the difficulties in cutting these heavy fabrics, and especially to the lack of support from the stitching on the $0^{\circ}$ face where only a bonding yarn maintains the fibres (Figure 3-b). The handling must participate also to the scattering of the results.

[Table 5 about here]

Let us remark that WEn OO 2 and WEn OO 3 exhibit a non-linear response (Figure 9). Since WEn OO 3 yields the largest pressures in the whole experiments, one can question whether the limits of the apparatus are reached, i.e. leaks may appear causing this non-linear response although there is no visible evidence of them. Another possible source of this non-linearity could be the capillary effects since WEn OO 3 is the only curve to tend downto 0 for decreasing fluid velocities. Eventually, as pointed out for instance by WU et al. for bi-directional stitched fiber mats [17], this could reveal the fabrics consolidation during the flow, most likely to appear for the highest pressures encountered. Further experiments must be carried out on these very heavy glass fibre fabrics. 


\subsubsection{Resistance curves for the Aeronautic $N C 2$}

Among the three $N C 2$ types tested, the most tricky one is Aeronautic, certainly because it is a quadriaxial material, the architecture of which is more complex (tortuous), but certainly also due to the very high homogeneity of the UD fabrics used to produce this $N C 2$. This is verified in Figure 10 where the limit injection rate, from which the tightness is no longer preserved, is only of the order of $4 \cdot 10^{-4} \mathrm{~m} / \mathrm{s}$ for the $O O$ faces, whereas for the Automotive and Wind Energy $N C 2$ the tightness remains perfect beyond fluid velocities of the order of $10^{-3} \mathrm{~m} / \mathrm{s}$.

\section{[Figure 10 about here]}

In these $N C 2$, again the $O O$ faces exhibit a larger resistance to the flow, as compared to the Automotive $N C 2$, along with a significant discrepancy (Table 6). Even if individually, the linear approximation remains founded for both faces (error [3.3-5.6] \% face $Z Z$ and $[2.7-7.4] \%$ face $O O)$, globally the results are quite scattered for the $O O$ face with a $34 \%$ mean distance to the linearity, but remain homogeneous for the $Z Z$ faces with a $8 \%$ error.

[Table 6 about here]

\subsection{Transverse permeability}

In the previous section, it was checked for the three types of $N C 2$ tested that the DARCY's approach was usable since a linear relationship was verified between the pressure drop and the fluid velocity. Indeed, the average (arithmetic) distance to the individual linearity is $5.6 \%$ and $2.5 \%$ for Automotive face $Z Z$ and $O O, 2.5 \%$ and $8 \%$ for Wind Energy, and $4.4 \%$ and $5.7 \%$ for Aeronautic. Now, from these individual linear regressions applied to every resistance curve (Figure 7, Figure 9, and Figure 10), the apparent permeability can easily be extracted as the inverse of the slope of these regressions.

\subsubsection{Permeability measured}

The permeability measurement results are reported in Table 7. It is verified, as can be expected from the resistance curves, that both permeabilities are relatively close 
for Automotive (4.46E-13/8.62E-13) and differ perceptibly for Wind Energy (9.96E13/2.40E-13) and Aeronautic (10.6E-13/1.87E-13). It seems that the permeability differential is proportional to the fibre volume fraction (Table 7 ), but also to the $N C 2$ weight (Figure 11) which presents the same trend due to the compaction mechanism used in our setup. As for the influence of stacking sequence on the permeability, it seems that no straight conclusion can be drawn from the present analysis (see face orientation in Figure 11).

[Table 7 about here]

[Figure 11 about here]

In these permeability results, the coefficient of variation measures the scattering of the individual permeabilities with respect to the average global permeability. As can be expected from the resistance curves, the lowest scattering around the average permeability is achieved for Automotive ( $3 \%$ for $Z Z$ face), the highest being for Wind Energy (14.7\% for ZZ face). But globally these variations are reasonable, considering for instance that LUNDSTRÖM et al. [32] present a $15 \%$ repeatability for some longitudinal permeabilities measured over some $300 \mathrm{~mm}$ long flows. The authors state that this repeatability variation is imputable to the fabric handling. Eventually, this handling may introduce the largest discrepancies that can be found in our experiments, and cannot therefore be easily avoided.

\subsubsection{Discussion on the permeability measurements}

To conclude these permeability measurements, it would be interesting to compare the values obtained with some from the literature. Unfortunately, the $N C 2$ transverse permeability has not been studied so far on other experimental setups. The multiaxial reinforcements displaying a meso-structure close enough to the $N C 2$ meso-structure are the $N C F$, even if their least homogeneity must strongly influence the permeability. Moreover, the versatility of the multiaxial reinforcements is so large (materials, stacking sequence, stitching pattern, ...) that it is unlikely that corresponding configurations can be found. However, we know that NCF effective plane permeabilities are of the order of $10^{-11}$ to $10^{-12} \mathrm{~m}^{2}$ ([32] for instance) and that this permeability can vary by a factor of 3 due to small variations of the fabric geometry [33]. In the present work, the stability and repeatability of the measurements, along with the homogeneity of 
the results of the order of $10^{-13}-10^{-12} \mathrm{~m}^{2}$, guaranty the validity of the transverse permeability measurements in saturated regime.

One of the most important feature of the $N C 2$ tested is the permeability differential which cannot be properly understood only through a macroscopic approach. As demonstrated experimentally and numerically by DRAPIER et al. [1], the stitching density plays a major role in the transverse permeability of the $N C 2$. The main conclusion from this work is that the only open-pores permitting the fluid to flow across the dense fabrics are the stitching holes. Following this conclusion, a morphological study of the stitching holes in the different $N C 2$ is presented below which could bring the first elements for some qualitative explanations for this permeability differential.

\section{Morphological characterization of the NC2 mul- tiaxial fabrics before and after experiment}

The three types of $N C 2$ material used to assess the transverse permeability, which were off-shelves, were first considered in this morphological investigation. Subsequently, further materials were considered here to draw some qualitative conclusions. Let us recall that the stitching density is similar for all these multiaxial reinforcements, with a $5 \mathrm{~mm} \times 5 \mathrm{~mm}$ stitching pattern.

\subsection{Measurement technique}

The idea of this morphology characterization is to try and relate the permeability differential to the stitching hole morphology. The stitching hole surfaces were first estimated by light transmission on a Corema ${ }^{\circledR}$ tridimensionnal measuring machine, Detec Vision type, injecting a diascopic natural light measured by a camera on the other face of the $N C 2$. A dedicated software then gives the corresponding surfaces (Figure 12-a). This technique applies only to carbon fibres since glass fibres will conduct the light, but more importantly transmission measures will give the minimum stitching hole surface common on both faces (Figure 13), and therefore will not give access to the differential in surface on both faces if it exists.

[Figure 12 about here] 
[Figure 13 about here]

Then we moved to measurements using the light reflection, with a special care brought to the ligthening of the carbon $N C 2$ which reflect very poorly the natural light. For these measurements a binocular magnifying glass was used, equipped with a $50 \mathrm{~mm}$ objective and indirect annular or direct lightening. Using the AnalySIS ${ }^{\circledR}$ software, the stitching hole surfaces were estimated by encircling manually on the pictures the visible space generated by the stitching yarn channel (Figure 12-b).

\subsection{Characterization before and after experience}

It appears that for the three $N C 2$ types tested previously, the stitching hole surface is of the same order for both faces before and after experiment (Table 8). Coefficients of variation are acceptable regarding the difficulty in defining the hole edge. It has to be noticed that for the Wind Energy $N C 2$, the $Z Z$ face cannot be characterized because $0^{\circ}$ fibres pass over the stitching yarns that are in the fibre direction.

[Table 8 about here]

The increase in the mean surface observed after experiment may explain the hysteretic behaviour such as observed in Figure 8 for decreasing fluid velocities. But the presented measures cannot on their own justify either the permeabilities, since surfaces are close for the three $N C 2$ types, or the permeability differential since both $O O$ and $Z Z$ surfaces are comparable.

\subsection{Influence of the stitching tension}

It is known that the stitching tension is of prime importance in forming the stitching hole, as sketched and seen in Figure 13. To assess this influence, the stitching hole surfaces were measured before and after experiments, on [+45/-45] carbon fibre $N C 2$, $534 \mathrm{~g} / \mathrm{m}^{2}$, with three different stitching tensions named 'normal', 'low', and 'very low'. The results are reported in Table 9.

[Table 9 about here] 
The measurements confirm that the stitching hole surface depends strongly on the stitching tension, as can be expected (see Figure 13) : when the stitching is loose, the stitching hole is the smallest before experiments and becomes the largest after experiments (Figure 14). This phenomenon can also be observed in NCF but is still more pronounced since the initial fabric is made with fibre tows which easily tend to bunch for increasing stitching tensions ([34], [35], [36]). Another information from

these results is that the lower the stitching tension, the higher the ratio $\frac{\bar{S}^{O O}}{\bar{S}^{Z Z}}$ before experiment. Even if this ratio of 0.67 for normal tension could be related to the ratio $\frac{\bar{k}_{Z Z}}{k_{O O}} \simeq 0.5$, this no longer holds after experiments where the ratio of the mean surfaces tends towards 1 for any tension.

These measurements demonstrate that the stitching tension will influence the stitching hole surface, in the sense that it will allow a motion of the fibres around the holes in which the fluid will flow. But it cannot explain the permeability differential observed in the permeability measurements. Eventually, the reconstruction of a complete stitching hole was attempted, in order to propose an explanation to this feature.

\subsection{Stitching hole $3 \mathrm{D}$ reconstruction}

Starting from the simplest $N C 2$, a biaxial Automotive, a plate made up of a single $N C 2$ was manufactured. After, cooling some successive polishings were made in the $90^{\circ}$ direction as represented in Figure 15.

[Figure 15 about here]

From the faces polished, some pictures were taken and the hole dimensions were measured. Then the 3D hole was reconstructed, as pictured in Figure 16. Since the stitching yarn will separate the $0^{\circ}$ face at the stitching hole place (see Figure 15), only the $90^{\circ}$ fibres are visible here.

[Figure 16 about here]

It can be seen that the $O O$ face is more wide-mouthed. This is in accordance with the permeability measurements which give $\frac{\bar{k}_{Z Z}}{k_{O O}} \simeq 0.5$. This geometry could well in turn result in an higher apparent permeability, but some fluid-structure simulations have to be carried out on this geometry to evaluate quantitatively this effect. 


\subsection{Conclusion on the morphological measurements}

In order to propose an explanation to the permeability differential demonstrated by the experiments, a morphological study has been carried out. The stitching hole surface measurements cannot, on their own, explain this differential since for the three $N C 2$ studied the average surfaces are very close for both faces, and remain close although a slight increase is verified after the permeability characterization experiments. This is confirmed on other $[+45,-45]$ biaxial $N C 2$ with varying stitching tensions, even if it has been shown that the higher the stitching tension, the larger the stitching hole surface increase.

Eventually, a quantitative reconstruction of stitching hole in 3D has been proposed. Clearly, the morphology of this hole is totally different on both faces, with a widemouthed shape on the $O O$ face, and simply a space between the $0^{\circ}$ fibres on the $Z Z$ face. Some numerical simulations should be carried out using a simplified geometry of this type to evaluate more precisely its effect on the resulting flow. Beyond this, obviously the stacking sequences and the constitutive material will modify the mesoscopical architecture, and then the role of the stitching hole geometry may be of the second order. Only a more detailed study at the ply level, similar to the one from BACKHOUSE [36] carried out on $N C F$ reinforcements, could bring relevant pieces of answer. To go farther, a multi-scale morphological study has to be considered as demonstrated by LEE and co-workers [10][14] or BinÉTRUY and co-workers [16].

\section{Conclusion}

In order to improve the composite solutions 'dry multiaxial reinforcements/ infusionbased processes', the transverse permeability has been studied for three types of $N C 2$, namely Automotive, Wind Energy and Aeronautic. Assuming classically that the transverse direction is a principal direction of the fabrics, the permeability is studied in saturated regime, from a macroscopical point of view. Relying on the Darcy's approach, knowing at the same time the gradient of pressure in the fluid and its velocity, the permeability is easily deduced.

A specific apparatus developed in DRAPIER et al. [1] was used, in which the specimens to be tested are maintained between honeycomb plates. A test fluid is then injected at controlled rate across the specimen, and both pressures before and after the specimen are recorded. This setup had to be modified to sustain the very high pressures induced 
by the $N C 2$ tested, especially the heaviest ones (Wind Energy and Aeronautic). The resistance curves plotted for the three types of $N C 2$ tested exhibit both a definite linearity and a good reproducibility. The use of the Darcy' law is then legitimate, and the permeabilities were identified from these curves.

Permeabilities measured are of the order of $10^{-13} \mathrm{~m}^{2}$. These permeabilities appear to depend on the material tested, but more surprisingly, they depend also on the face receiving the fluid, with a ratio that can go from 0.5 down to 0.17 when the $N C 2$ weight increases. This permeability differential was observed for the three types of $N C 2$, and it was shown to be reversible and reproducible. This original feature had never been reported in the literature before. The whole set of permeabilities is homogeneous with a repeatability around $15 \%$. This sensitivity must be related either to the lack of support for the $0^{\circ}$ fibres, or to the very high homogeneity of the fabrics that make up the $N C 2$, or more certainly to the handling of the specimens.

An attempt was made to propose an explanation to the permeability differential. Following a conclusion from DRAPIER et al. [1], we tried to relate the stitching holes morphology to the permeability variations. Unfortunately, the superficial measures carried out using the light reflection were not able to explain either the permeability variations between the materials or the permeability differential for the same material. It was also demonstrated that the stitching tension would participate in modifying the stitching hole, since the stitching yarn maintains the fibres around the holes during the fluid flow. Eventually a 3D reconstruction of a stitching hole was attempted which showed that the 3D morphology could yield some explanations to the permeability differential. This has to be validated by numerical simulations and deepened through multiscale analyses.

At the moment, the knowledge brought by these measurements of the $N C 2$ transverse permeability should lead to a better understanding in the manufacturing cycles. Especially, the processing parameters should take advantage of the permeability differential to modify, for equal mechanical properties, the manufacturing cycles. Industrial applications require now further measurements. For the future, the testing device has to be redesigned to ensure a sufficient tightness while allowing the control of the fibre volume fraction, in order to get closer to the industrial manufacturing conditions. Also, the assessment of the transverse transient permeability should be considered. 


\section{Acknowledgements}

The authors wish to acknowledge the Rhône-Alpes Region for its support to this work through the programme 'Thématique Prioritaire 2000-2002'.

\section{References}

[1] S. Drapier, A. Pagot, A. Vautrin, and P. Henrat. Influence of the stitching density on the transverse permeability of non-crimped new concept $(N C 2)$ multiaxial reinforcements : measurements and predictions. Comp. Sci. Technol, 62(15):19791991, 2002.

[2] A.J. Ahn and J.C. Seferis. Autoclave resin infusion process : analysis and prediction of content. Sampe Quarterly, 21(2):3-10, 1990.

[3] S.C. Joshi, L. Xiao-Lin, Y.C. Lam, and J. Sheridam. Simulation of resin film infusion process using finite element/nodal control volume approach. Adv. Compos. Letters, 8(3):101-104, 1999.

[4] C. Williams, J. Summerscales, and S. Grove. Resin infusion under flexible tooling (RIFT): A review. Composites/Part A: Applied Sci. and Manufacturing, pages 517-524, 1996.

[5] D.C. Blest, S. Mckee, A.K. Zulkifle, and P. Marshall. Curing simulation by autoclave resin infusion. Comp. Sci. Technol, 59:2297-2313, 1999.

[6] A.C. Caba, R.B. Rattazzi, and A.C. Loos. Verification of a simulation model for resin film infusion of complex shaped composite structures. J. Reinf. Plast. Compos, 18(16):1465-1477, 1999.

[7] M. Kaviany. Principles of heat transfer in porous media (2 ${ }^{\text {nd }}$ edition). SpringerVerlag New York, Inc, 1995.

[8] J. Bear. Dynamics of fluids in porous media. Number ISBN-0-486-65675-6. Dover Publications, Inc., 1988.

[9] K.L. Adams, B. Miller, and L. Rebenfeld. Forced in-plane flow of an epoxy resin in fibrous networks. Polym. Eng. Sci, 26(20):1434-1441, 1986. 
[10] C. H. Shih and L.J. Lee. Effect of fiber architecture on permeability in liquid composite molding. Polym. Compos, 19(5):626-639, 1998.

[11] N. Takano, M. Zako, T. Yoshioka, and K. Terada. Homogenization method to predict three-dimensional permeabilities considering micro-macro and solid-fluid interactions. In A. Vautrin and T. Massart, editors, Proceedings of ICCM12, Paris 5-7 July, 1999.

[12] Y. Henzel, J. Bréard, F. Trochu, and R. Blanlot. A standard characterization of saturated and unsaturated flow behaviours in porous media. In A. Vautrin and T. Massart, editors, Proceedings of ICCM12, Paris 5-7 July, 1999.

[13] V. P. Stavrov, E.I. Kremenevskaya, V.V. Stavrov, and V.M. Tkachev. Effect of the structure of a fibrous layer on its permeability for a nonlinearly viscous fluid. Mech. of Comp. Mat., 33(4):554 - 563, July - August 1997.

[14] B. Yu, L. J. Lee, and H. Cao. A fractal in-plane permeability model for fabrics. Polym. Compos., 23(2):201-221, 2002.

[15] H. Darcy. Les Fontaines Publiques de la ville de Dijon. Dalmont, Paris, 1856.

[16] C. Binétruy, B. Hilaire, and J. Pabiot. The interactions between flows occurring inside and outside fabric tows during RTM. Comp. Sci. Technol, 57:587-596, 1997.

[17] C.-H. Wu, T.J. Wang, and L. J. Lee. Trans-plane fluid permeability measurement and its application in liquid composite molding. Polym. Compos., 15(4):289-298, 1994.

[18] M.A.A. Spaid and F.R. Jr Phelan. Modeling void formation dynamics in fibrous porous media with the lattice boltzmann method. Composites/Part A, 29A:749755, 1999.

[19] F.R. Jr Phelan and G. Wise. Analysis of transverse flow in aligned fibrous porous media. Traduit de Mekhanik Kompozitnykh Materialov, 27:25-34, 1995.

[20] T.A.K. Sadiq, S.G. Advani, and R.S. Parnas. Experimental investigation of transverse flow through aligned cylinders. Int. J. Multiphase Flow, 21(5):755-774, 1995.

[21] R.S. Parnas, A.J. Salem, T.A.K. Sadiq, H.P. Wang, and S.G. Advani. The interaction between micro- and macro-scopic flow in RTM preforms. Compos. Struct, 27:93-107, 1994. 
[22] C. Binétruy and J. Pabiot. Effects of fabric architectural heterogeneities on effective and saturated permeabilities in RTM. In A. Vautrin and T. Massart, editors, Proceedings of ICCM12, Paris 5-7 July, 1999.

[23] J.G. Williams, C.E. Morris, and B.C. Ennis. Liquid flow through aligned fiber beds. Polym. Eng. Sci., 14(6):413-419, 1974.

[24] Y. Ma and R. Shishoo. Permeability characterization of different architectural fabrics. J. Comp. Mat, 33(8):729-750, 1999.

[25] T.S. Lundström, R. Stenberg, R. Bergström, H. Partanen, and P.A. Birkeland. Inplane permeability measurements: a nordic round-robin study. Composites/Part A: Applied Sci. and Manufacturing, 31:29-43, 2000.

[26] V.H. Hammond and A.C. Loos. The effects of fluid type and viscosity on steadystate and advancing front permeability behavior of textile preforms. J. Reinf. Plast. Compos., 16(1):50-72, 1997.

[27] W.O. Ballata and S.M. Walsh. Determination of the transverse permeability of a fiber preform. J. Reinf. Plast. Compos, 18(16):1450-1464, 1999.

[28] T.L. Luce, S.G. Advani, G. Howard, J, and R.S. Parnas. Permeability characterization. Part 2 : flow behavior in multi-layer preforms. Polym. Compos, 16(6):446-458, 1995.

[29] L. Trevino, K. Rupel, W.B. Young, M.J. Liou, and L.J. Lee. Analysis of resin injection molding in molds with preplaced fiber mats. 1: Permeability and compressibility measurements. Polym. Compos, 12(1):20-29, 1991.

[30] J. Bréard, A. Saouab, and G. Bouquet. Mesure de la perméabilité spatiale d'un renfort tridimensionnel pour matériaux composites a matrice polymere. European Phys. Journal Applied, 1:269-278, 1998.

[31] S.H. Ahn, W.I. Lee, and G.S. Springer. Measurement of the three-dimensional permeability of fiber preforms using embedded fiber optic sensors. J. Comp. Mat, 29(6):714-733, 1995.

[32] T.S. Lundström, B.R. Gebart, and E. Sandlund. In-plane permeability measurements on fiber reinforcements by the multi-cavity parallel. Polym. Compos, 20(1):146-154, 1999. 
[33] T.S. Lundström. The permeability of non-crimp stitched fabrics. Composites/Part A: Applied Sci. and Manufacturing, 31:1345-1353, 2000.

[34] S. Drapier and M.R. Wisnom. Finite-element investigation of the compressive strength of non-crimp-fabric-based composites. Comp. Sci. Technol., 59(12):12871297, 1999.

[35] S. Drapier and M.R. Wisnom. Finite-element investigation of the interlaminar shear strength of non-crimp-fabric-based composites. Comp. Sci. Technol., 59(16):2351-2362, 1999.

[36] R. Backhouse. Multiaxial non-crimp fabrics: characterisation of manufacturing capability for composite aircraft structure applications. PhD thesis, Cranfield University, School of Industrial and Manufacturing Science, May 1998. 


\section{List of Tables}

1 Multiaxial $N C 2$ characteristics. . . . . . . . . . . . . . 25

2 Repeatability of the $N C 2$ Automobile [0/90] fabrics corresponding to Figure 6. . . . . . . . . . . . . . . . 26

3 Estimation of the $N C 2$ thickness, and corresponding fibre volume fractions. . . . . . . . . . . . . . . . . . . 26

4 Estimate and repeatability of the measurements for the $\mathrm{NC} 2$ Automotive $[0 / 90]\left(430 \mathrm{~g} / \mathrm{m}^{2}\right)$ fabrics corresponding to Figure $7 \ldots \ldots 26$

5 Estimate and repeatability of the measurements for the NC2 Wind energy $[-45 /+45 / 0]\left(975 \mathrm{~g} / \mathrm{m}^{2}\right)$ corresponding to Figure $9 . \ldots \ldots 27$

6 Estimate and repeatability of the measurements for the $\mathrm{NC} 2$ Aeronautic $[-45 / 0 /+45 / 90]\left(600 \mathrm{~g} / \mathrm{m}^{2}\right)$ corresponding to Figure 10 . . . . . . . . 27

7 Saturated transverse permeabilities identified on the three $N C 2$ types considered. . . . . . . . . . . . . . . . . . . . 28

8 Stitching hole surfaces of multiaxial NC2 BEFORE and AFTER experiment. . . . . . . . . . . . . . . . . . 28

$9 \quad$ Stitching hole surfaces of multiaxial $N C 2$ BEFORE and AFTER experiment for various tensions in $[+45 /-45]\left(534 \mathrm{~g} / \mathrm{m}^{2}\right) \mathrm{NC} 2 \ldots . . .$. . 


\begin{tabular}{|c|c|c|c|}
\hline Application type & $\begin{array}{c}\text { Automotive } \\
430 \mathrm{~g} / \mathrm{m}^{2}\end{array}$ & $\begin{array}{l}\text { Wind energy } \\
\qquad 975 \mathrm{~g} / \mathrm{m}^{2}\end{array}$ & $\begin{array}{l}\text { Aeronautics } \\
600 \mathrm{~g} / \mathrm{m}^{2}\end{array}$ \\
\hline Stacking sequence & {$[0 / 90]$} & {$[-45 /+45 / 0]$} & {$[-45 / 0 /+45 / 90]$} \\
\hline $\begin{array}{l}\text { Superficial weight } \\
m_{s}\left(g / m^{2} / \text { ply }\right)\end{array}$ & $2 \times 215$ & $438 / 242 / 242^{2}$ & $4 \times 150$ \\
\hline Type of Plies & UD Plies & UD Plies with yarn & UD Plies with yarn \\
\hline Fibres & $\begin{array}{l}\text { Carbon Zoltek Panex } \\
33 \text { TW048-11 }\end{array}$ & $\begin{array}{l}\text { E Glass (Roving } \\
1200 \text { tex) }\end{array}$ & $\begin{array}{l}\text { Carbon Toray HR } \\
\text { T700 GC (1600 tex) }\end{array}$ \\
\hline $\begin{array}{l}\text { Filament diameter } \\
(\mu \mathrm{m})\end{array}$ & 7 & 9 & 7 \\
\hline $\begin{array}{l}\text { Fibre vol. mass } \rho_{f} \\
\left(\mathrm{~kg} / \mathrm{m}^{3}\right)\end{array}$ & 1,810 & 2,600 & 1,810 \\
\hline Stitching yarn & Polyamide & Polyamide or glass & Polyam. or carbon \\
\hline
\end{tabular}

Table 1: Multiaxial $N C 2$ characteristics.

\footnotetext{
${ }^{2}$ Wind Energy : total superficial mass $=$ fabric superficial mass + stitching yarn mass
} 


\begin{tabular}{|l||c|c|c||c|}
\hline & Auto 1 & Auto 2 & Auto 3 & Auto 1-2-3 \\
\hline \hline $\bar{E}(U)(\%)$ & 4.1 & 1.4 & 2.1 & 3.9 \\
\hline$E(U)_{\max }(\%)$ & 14.4 & 7.3 & 7.9 & 13.6 \\
\hline$E(U)_{\min }(\%)$ & 0.06 & $<0.01$ & 0.19 & 0.01 \\
\hline
\end{tabular}

Table 2: Repeatability of the NC2 Automobile [0/90] fabrics corresponding to Figure 6.

\begin{tabular}{|c|c|c|c|}
\hline Industrial application & Automotive & Wind energy & Aeronautics \\
\hline Initial thickness $h_{0 N}^{N C 2}(\mathrm{~mm})$ & 0.74 & 0.88 & 0.7 \\
\hline Compacted thickness $h_{3234 N}^{N C 2}(\mathrm{~mm})$ & 0.58 & 0.68 & 0.52 \\
\hline Fibre volume fraction $v_{f}=\frac{m_{s}}{\rho_{f} h}(\%)$ & 41 & 52 & 64 \\
\hline Equivalent compaction pressure (bars) & 2.7 & 2.9 & 3.3 \\
\hline
\end{tabular}

Table 3: Estimation of the $N C 2$ thickness, and corresponding fibre volume fractions.

\begin{tabular}{|ll||c||c|c|}
\hline & & $\bar{E}(U)(\%)$ & $E(U)_{\max }(\%)$ & $E(U)_{\min }(\%)$ \\
\hline \hline \multirow{2}{*}{ Face ZZ } & Auto ZZ 1 & 7.6 & 23.9 & 0.27 \\
\cline { 2 - 5 } & Auto ZZ 2 & 6.2 & 24.7 & 0.57 \\
\cline { 2 - 5 } & Auto ZZ 3 & 2.9 & 12.7 & 0.04 \\
\hline \hline \multirow{2}{*}{ Face OO } & Auto OO 1 & 4.1 & 14.4 & 0.06 \\
\cline { 2 - 5 } & Auto OO 2 & 1.4 & 7.3 & $<0.01$ \\
\hline \hline \multirow{2}{*}{ Face ZZ } & Auto OO 3 & 2.1 & 7.9 & 0.19 \\
\hline Face OO & Auto OO 1-2-3 & 3.9 & 13.6 & 0.01 \\
\hline
\end{tabular}

Table 4: Estimate and repeatability of the measurements for the NC2 Automotive [0/90] $\left(430 \mathrm{~g} / \mathrm{m}^{2}\right)$ fabrics corresponding to Figure 7 . 


\begin{tabular}{|cc||c||c|c|}
\hline & & $\bar{E}(U)(\%)$ & $E(U)_{\max }(\%)$ & $E(U)_{\min }(\%)$ \\
\hline \hline \multirow{2}{*}{ Face ZZ } & Wind Energy ZZ 1 & 4.1 & 13.5 & 0.1 \\
\cline { 2 - 5 } & Wind Energy ZZ 2 & 0.8 & 1.6 & 0.01 \\
\cline { 2 - 5 } & Wind Energy ZZ 3 & 2.7 & 10.4 & 0.04 \\
\hline \hline \multirow{2}{*}{ Face OO } & Wind Energy OO 1 & 3 & 12.7 & 0.12 \\
\cline { 2 - 5 } & Wind Energy OO 2 & 12.5 & 34.3 & 1.1 \\
\hline \hline \multirow{2}{*}{ Face ZZ } & Wind Energy OO 3 & 8.8 & 33.1 & 0.6 \\
\hline Face OO & Wind Energy ZZ 1-2-3 & 12.2 & 29.3 & 0.5 \\
\hline
\end{tabular}

Table 5: Estimate and repeatability of the measurements for the $N C 2$ Wind energy $[-45 /+45 / 0]\left(975 \mathrm{~g} / \mathrm{m}^{2}\right)$ corresponding to Figure 9 .

\begin{tabular}{|ll||c||c|c|}
\hline & & $\bar{E}(U)(\%)$ & $E(U)_{\max }(\%)$ & $E(U)_{\min }(\%)$ \\
\hline \hline \multirow{2}{*}{ Face ZZ } & Aero ZZ 1 & 5.8 & 16.8 & 0.4 \\
\cline { 2 - 5 } & Aero ZZ 2 & 3.3 & 5.6 & 0.07 \\
\cline { 2 - 5 } & Aero ZZ 3 & 4.1 & 13.2 & 1.7 \\
\hline \hline \multirow{2}{*}{ Face OO } & Aero OO 1 & 7.4 & 26.5 & 1.1 \\
\cline { 2 - 5 } & Aero OO 2 & 7.1 & 17.4 & 0.7 \\
\cline { 2 - 5 } & Aero OO 3 & 2.7 & 6.4 & 0.07 \\
\hline \hline \multirow{2}{*}{ Face ZZ } & Aero ZZ 1-2-3 & 8.1 & 24.5 & 0.8 \\
\hline Face OO & Aero OO 1-2-3 & 34.1 & 91.3 & 3.1 \\
\hline
\end{tabular}

Table 6: Estimate and repeatability of the measurements for the $N C 2$ Aeronautic [-45/0/+45/90] $\left(600 \mathrm{~g} / \mathrm{m}^{2}\right)$ corresponding to Figure 10. 


\begin{tabular}{|c|c|c|c|c|c|c|}
\hline $\begin{array}{l}\text { Multiaxial } \\
\text { Reinforcements }\end{array}$ & \multicolumn{2}{|c|}{$\begin{array}{l}\text { Automotive } \\
\qquad v_{f}=41 \%\end{array}$} & \multicolumn{2}{|c|}{$\begin{array}{c}\text { Wind Energy } \\
v_{f}=52 \%\end{array}$} & \multicolumn{2}{|c|}{$\begin{array}{c}\text { Aeronautic } \\
v_{f}=64 \%\end{array}$} \\
\hline Face & $Z Z$ & $O O$ & $Z Z$ & $O O$ & $Z Z$ & $O O$ \\
\hline Mean transverse perm. $\bar{k}\left(10^{-13} \mathrm{~m}^{2}\right)$ & 4.46 & 8.62 & 9.96 & 2.4 & 10.6 & 1.87 \\
\hline Coefficient of variation (\%) & 3.04 & 8.71 & 14.7 & 7.52 & 13.6 & 12.5 \\
\hline Ratio $\frac{\bar{k}_{Z Z}}{\bar{k}_{O O}}$ & \multicolumn{2}{|c|}{0.52} & \multicolumn{2}{|c|}{4.15} & \multicolumn{2}{|c|}{5.9} \\
\hline Ratio $\frac{\min \left(\bar{k}_{Z Z}, \bar{k}_{O O}\right)}{\max \left(\bar{k}_{Z Z}, \bar{k}_{O O}\right)}$ & \multicolumn{2}{|c|}{0.52} & \multicolumn{2}{|c|}{0.24} & \multicolumn{2}{|c|}{0.17} \\
\hline
\end{tabular}

Table 7: Saturated transverse permeabilities identified on the three $N C 2$ types considered.

\begin{tabular}{|c|c|c|c|c|c|c|c|}
\hline \multirow{2}{*}{\multicolumn{2}{|c|}{$\begin{array}{l}\text { Industrial applications } \\
\text { Face }\end{array}$}} & \multicolumn{2}{|c|}{ Automotive } & \multicolumn{2}{|c|}{ Wind energy } & \multicolumn{2}{|c|}{ Aeronautic } \\
\hline & & $O O$ & $Z Z$ & $O O$ & $Z Z$ & $O O$ & $Z Z$ \\
\hline \multirow{2}{*}{$\begin{array}{l}\text { BEFORE } \\
\text { experiment }\end{array}$} & Avg. stitch. hole surf. $\bar{S}\left(m^{2}\right)$ & 0.05 & 0.05 & 0.07 & - & 0.05 & 0.05 \\
\hline & Coef. of variation & 0.4 & 0.14 & 0.19 & - & 0.34 & 0.14 \\
\hline \multirow{2}{*}{$\begin{array}{l}\text { AFTER } \\
\text { experiment }\end{array}$} & Avg. stitch. hole surf. $\bar{S}\left(\mathrm{~mm}^{2}\right)$ & 0.135 & 0.14 & 0.13 & - & 0.146 & 0.138 \\
\hline & Coef. of variation & 0.23 & 0.19 & 0.19 & - & 0.25 & 0.25 \\
\hline
\end{tabular}

Table 8: Stitching hole surfaces of multiaxial NC2 BEFORE and AFTER experiment. 


\begin{tabular}{|c|c|c|c|c|c|c|c|}
\hline \multirow{2}{*}{\multicolumn{2}{|c|}{$\begin{array}{r}\text { Stitching tension } \\
\text { Face }\end{array}$}} & \multicolumn{2}{|c|}{ Normal } & \multicolumn{2}{|c|}{ Low } & \multicolumn{2}{|c|}{ Very low } \\
\hline & & $O O$ & $Z Z$ & $O O$ & $Z Z$ & $O O$ & $Z Z$ \\
\hline \multirow{3}{*}{$\begin{array}{l}\text { BEFORE } \\
\text { experiment }\end{array}$} & Avg. stitch. hole surf. $\bar{S}\left(m m^{2}\right)$ & 0.098 & 0.066 & 0.072 & 0.069 & 0.054 & 0.058 \\
\hline & Coef. of variation & 0.31 & 0.2 & 0.37 & 0.25 & 0.32 & 0.18 \\
\hline & $\bar{S}_{O O} / \bar{S}_{Z Z}$ & \multicolumn{2}{|c|}{0.67} & \multicolumn{2}{|c|}{0.94} & \multicolumn{2}{|c|}{1.07} \\
\hline \multirow{3}{*}{$\begin{array}{l}\text { AFTER } \\
\text { experiment }\end{array}$} & Avg. stitch. hole surf. $\bar{S}\left(\mathrm{~mm}^{2}\right)$ & 0.137 & 0.146 & 0.16 & 0.17 & 0.174 & 0.181 \\
\hline & Coef. of variation & 0.19 & 0.15 & 0.19 & 0.15 & 0.06 & 0.19 \\
\hline & $\bar{S}_{O O} / \bar{S}_{Z Z}$ & \multicolumn{2}{|c|}{1.06} & \multicolumn{2}{|c|}{1.06} & \multicolumn{2}{|c|}{1.04} \\
\hline
\end{tabular}

Table 9: Stitching hole surfaces of multiaxial NC2 BEFORE and AFTER experiment for various tensions in $[+45 /-45]\left(534 \mathrm{~g} / \mathrm{m}^{2}\right) \mathrm{NC} 2$. 


\section{List of Figures}

1 Schematic of composite manufacturing with Resin Infusion Processes : (a) stacking, (b) infusion of the resin in the preforms, and (c) curing. . 31

2 Principle of the transverse permeability measurement. . . . . . . . . . . 31

$3 \quad \mathrm{NC} 2$ multiaxial fabrics : (a) face denoted subsequently by ZZ and (b) face denoted subsequently by OO . . . . . . . . . . . . . . . 32

4 Through the thickness permeability measurement fixture developed in [1]. 33

5 Protocol of the saturated transverse permeability measurement. . . . . 34

6 Resistance curves for Automotive $N C 2$ : linearity and repeatability of the resistance curve. . . . . . . . . . . . . . . . . . . . 35

$7 \quad$ Resistance curves for Automotive $N C 2 . \quad \ldots \ldots$. . . . . . . . . 36

8 Resistance curves for Automotive $N C 2$ : inverse process and linear fit of the permeability. . . . . . . . . . . . . . . . 37

$9 \quad$ Resistance curves for Wind Energy NC2. . . . . . . . . . . . . . 38

10 Resistance curves for Aeronautic NC2. . . . . . . . . . . . . . . . . 39

11 Transverse saturated permeabilities as functions of the $N C 2$ multiaxial weight (corresponding fibre orientation indicated between brackets). . . 40

12 Example of stitching hole measurement surfaces in (a) transmission for Aeronautic $N C 2$ and (b) reflection for Wind Energy $N C 2 . \quad \ldots . . . .40$

13 Conical shape of the stitching hole due to the stitching tension in biaxial Automotive $N C 2 . \ldots \ldots \ldots 4 . \ldots \ldots$

14 Stitching hole surface measurements in [+45/-45] NC2 BEFORE and AFTER permeability assessment experiments for varying stitching tension. 41

15 3D reconstruction of a stitching hole in Automotive [0/90] NC2 . . . . 42

$163 \mathrm{D}$ reconstruction of a stitching hole in Automotive [0/90] $N C 2$ : view (a) from the zig-zag face and (b) from the side. . . . . . . . . . . . . 43 


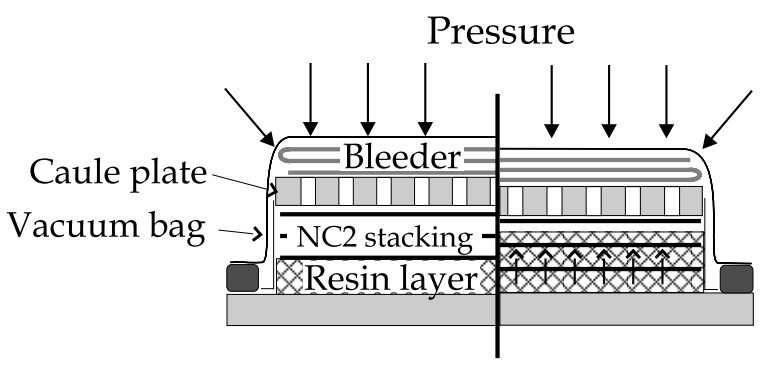

(a)

(b)

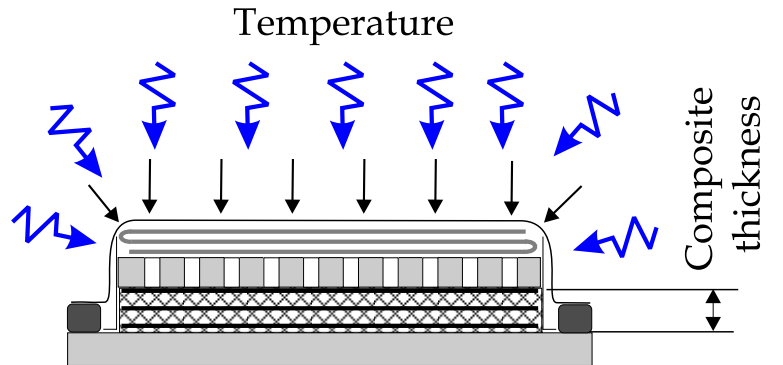

(c)

Figure 1: Schematic of composite manufacturing with Resin Infusion Processes : (a) stacking, (b) infusion of the resin in the preforms, and (c) curing.

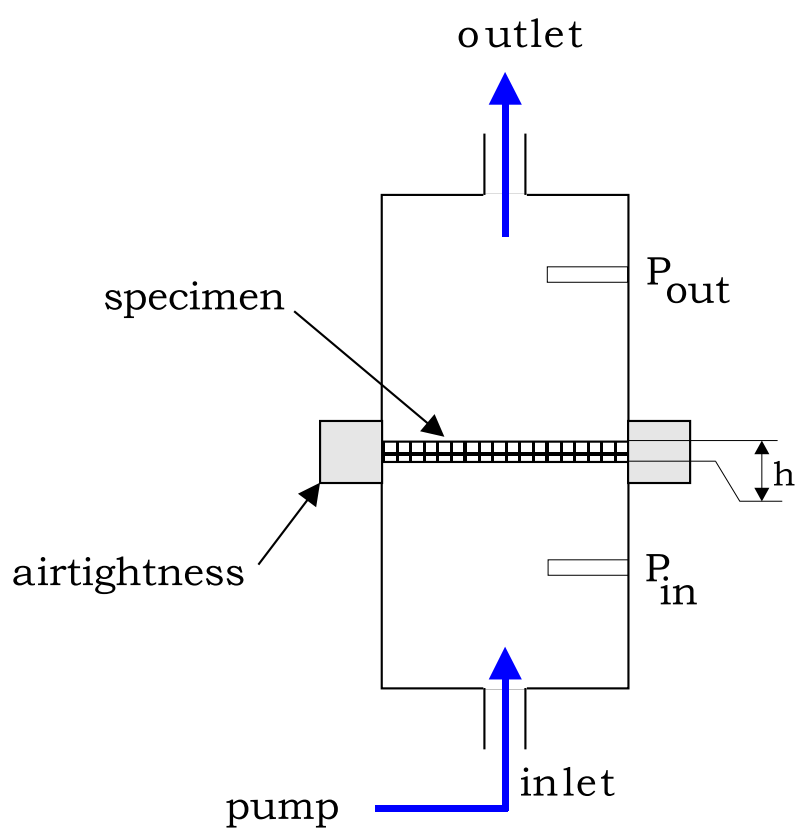

Figure 2: Principle of the transverse permeability measurement. 


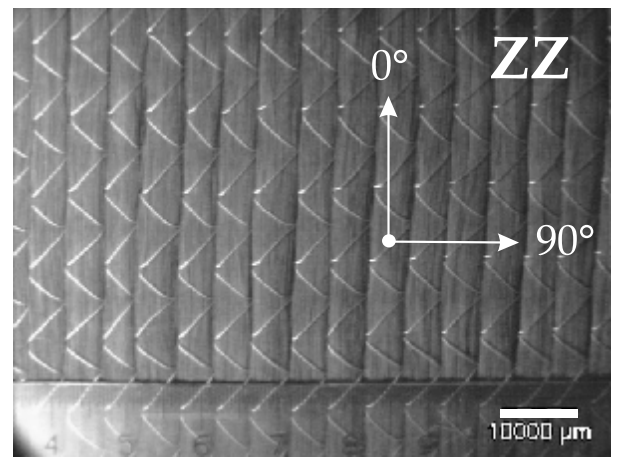

(a)

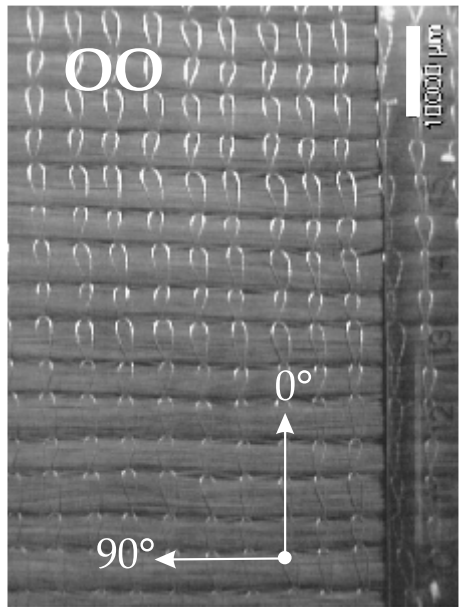

(b)

Automotive [0/90]

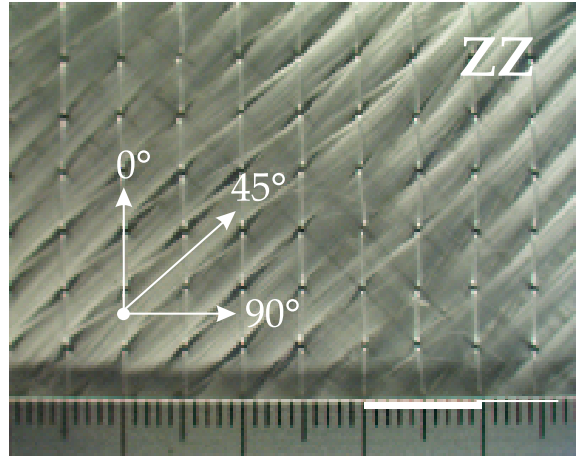

$10 \mathrm{~mm}$

(a)

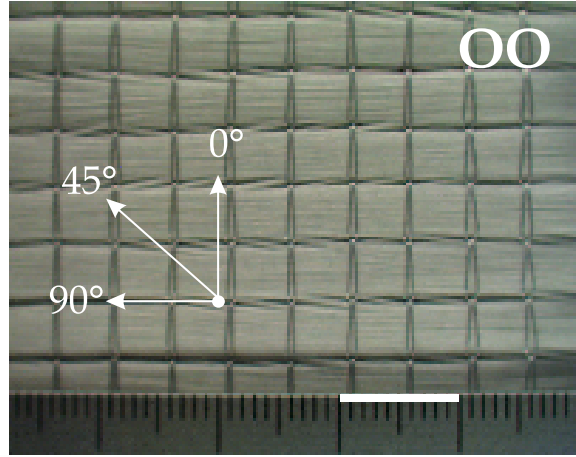

$10 \mathrm{~mm}$

(b)

Wind energy $[90 /-45 /+45]$

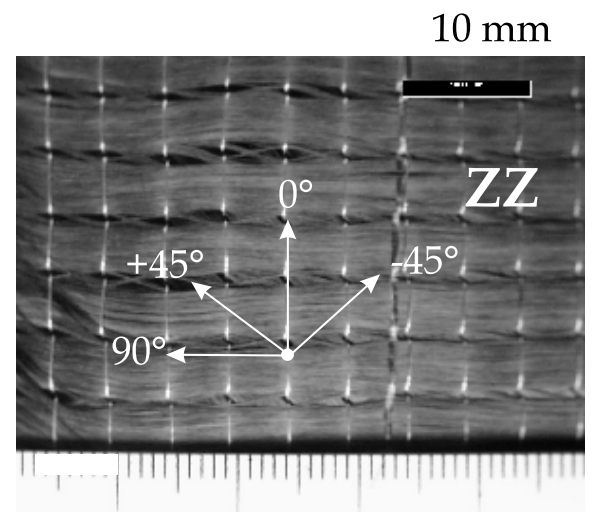

(a)

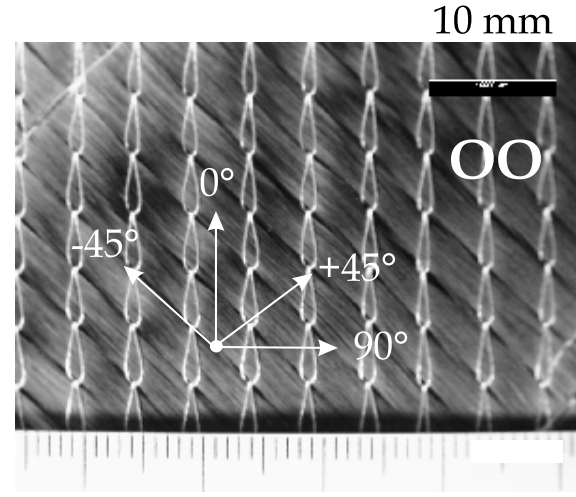

(b)

$$
\text { Aeronautic }[-45 / 0 /+45 / 90]
$$

Figure 3: NC2 multiaxial fabrics : (a) façe denoted subsequently by ZZ and (b) face denoted subsequently by OO. 


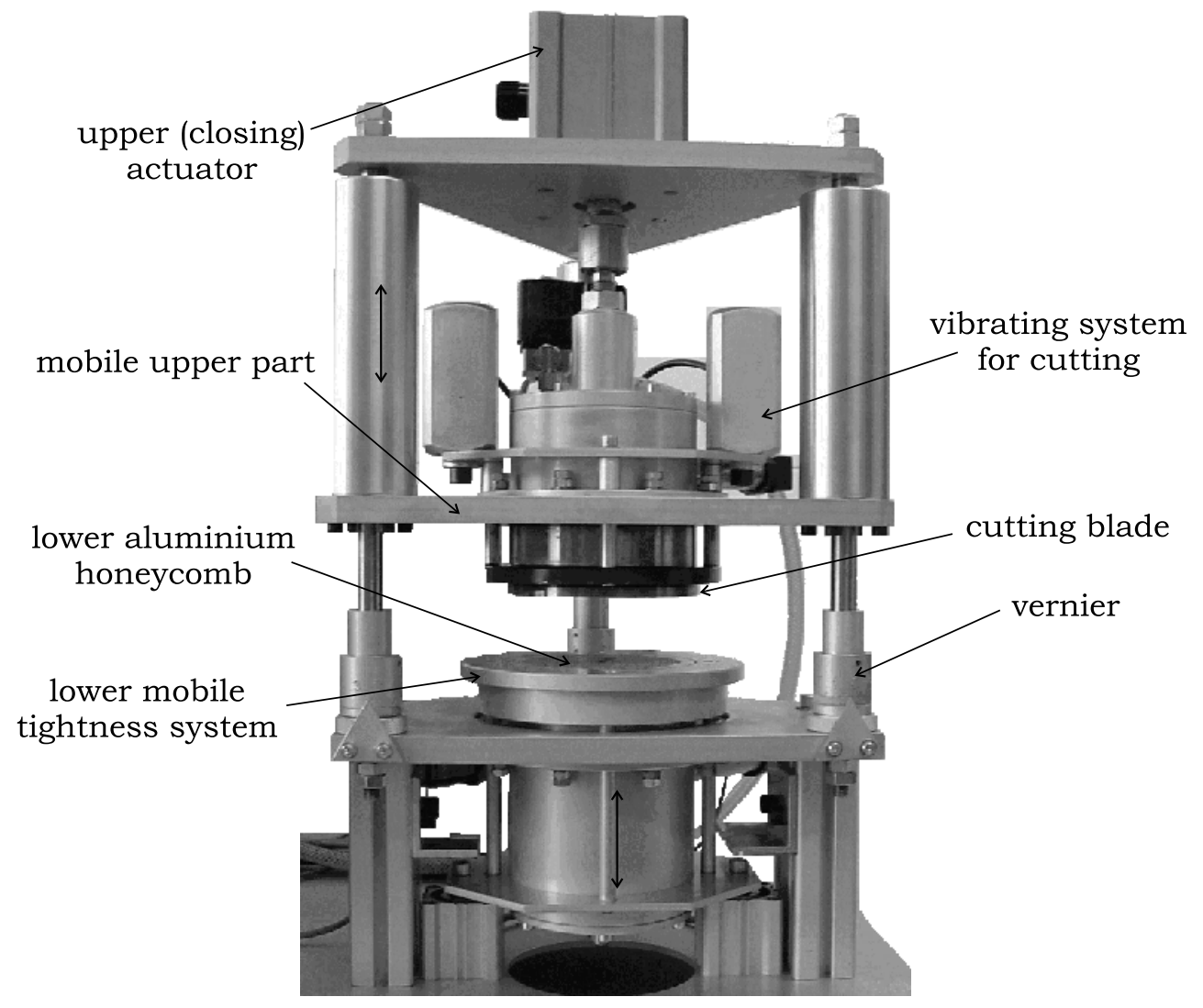

Figure 4: Through the thickness permeability measurement fixture developed in [1]. 


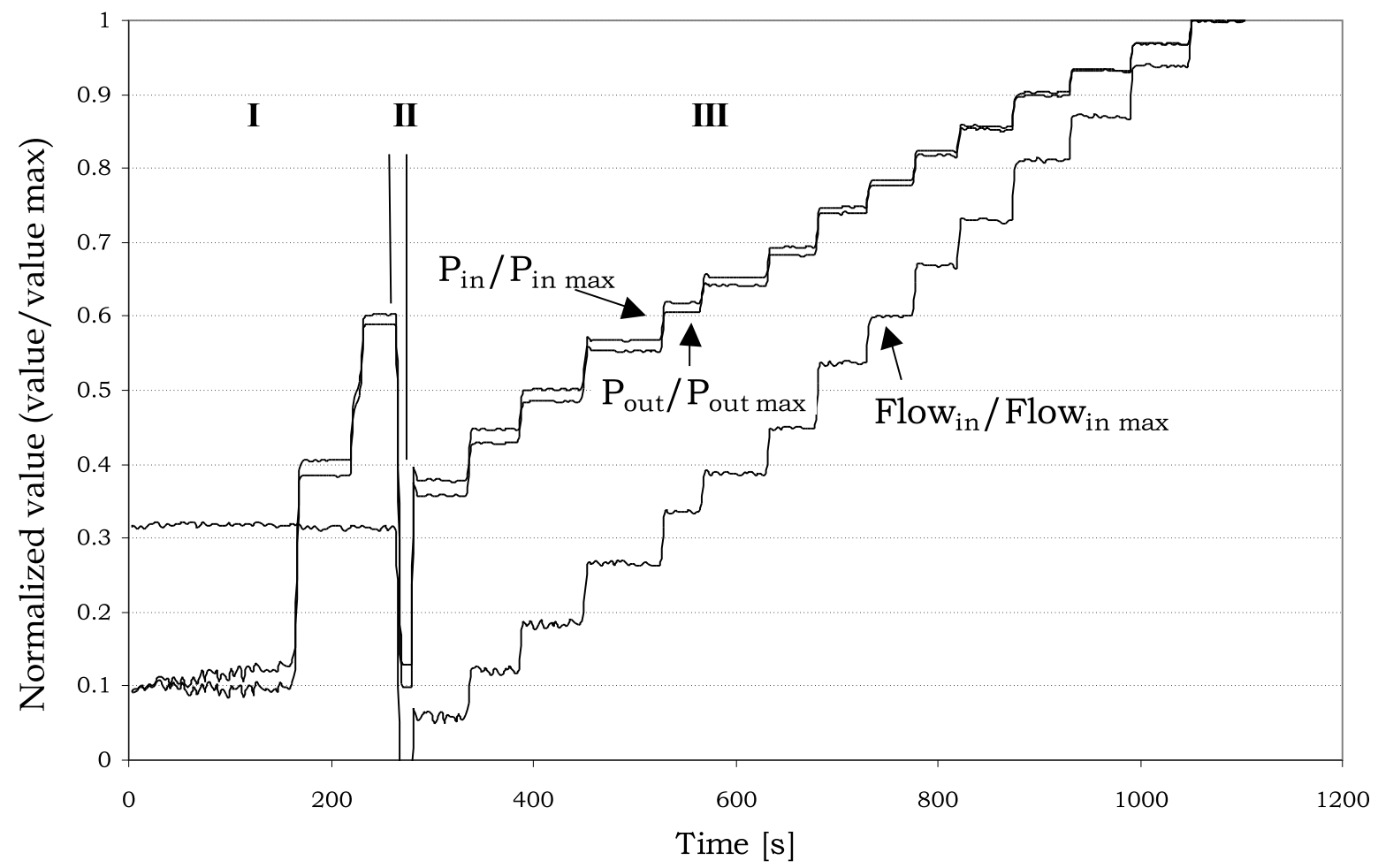

Figure 5: Protocol of the saturated transverse permeability measurement. 


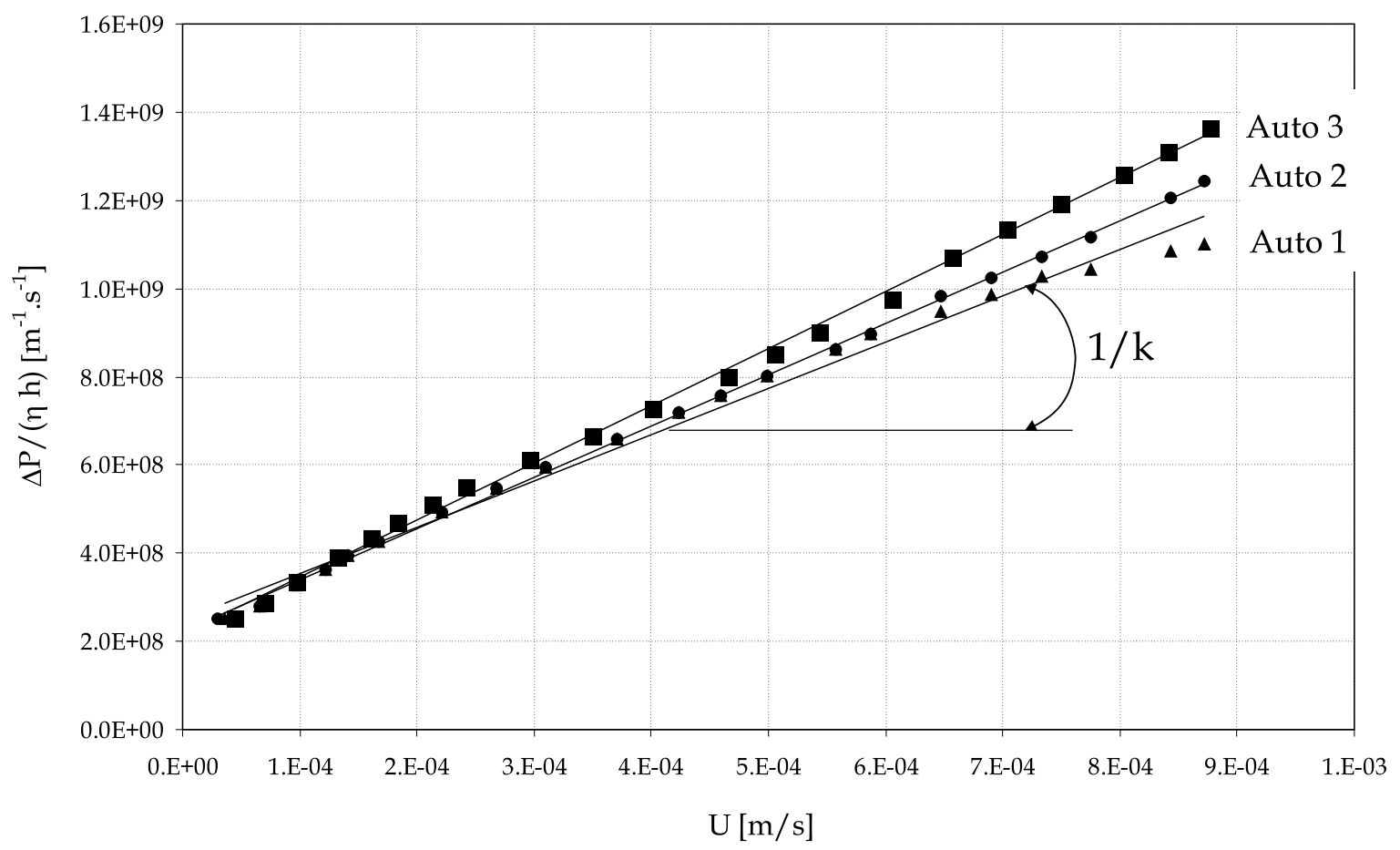

Figure 6: Resistance curves for Automotive $N C 2$ : linearity and repeatability of the resistance curve. 


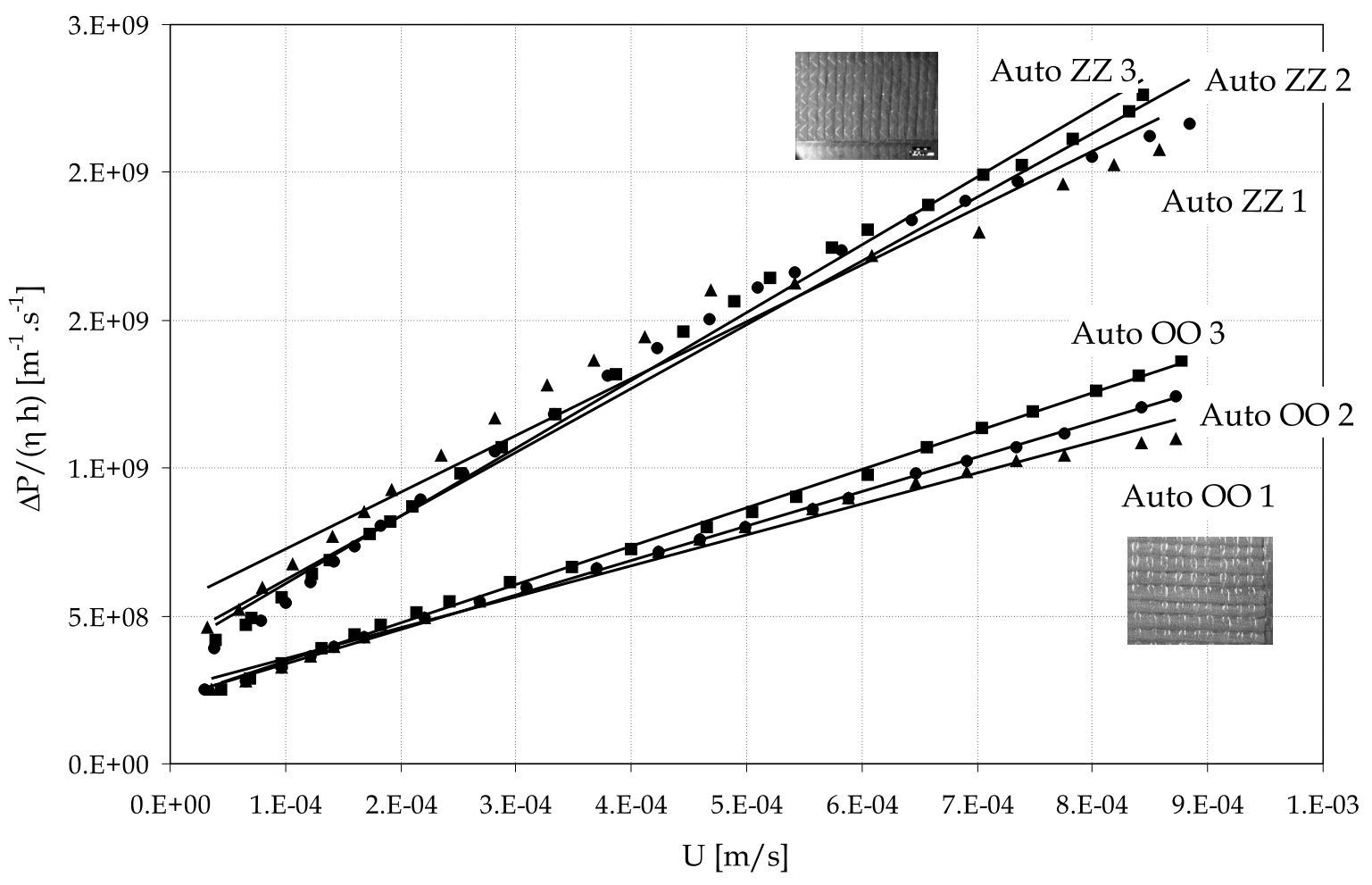

Figure 7: Resistance curves for Automotive $N C 2$. 


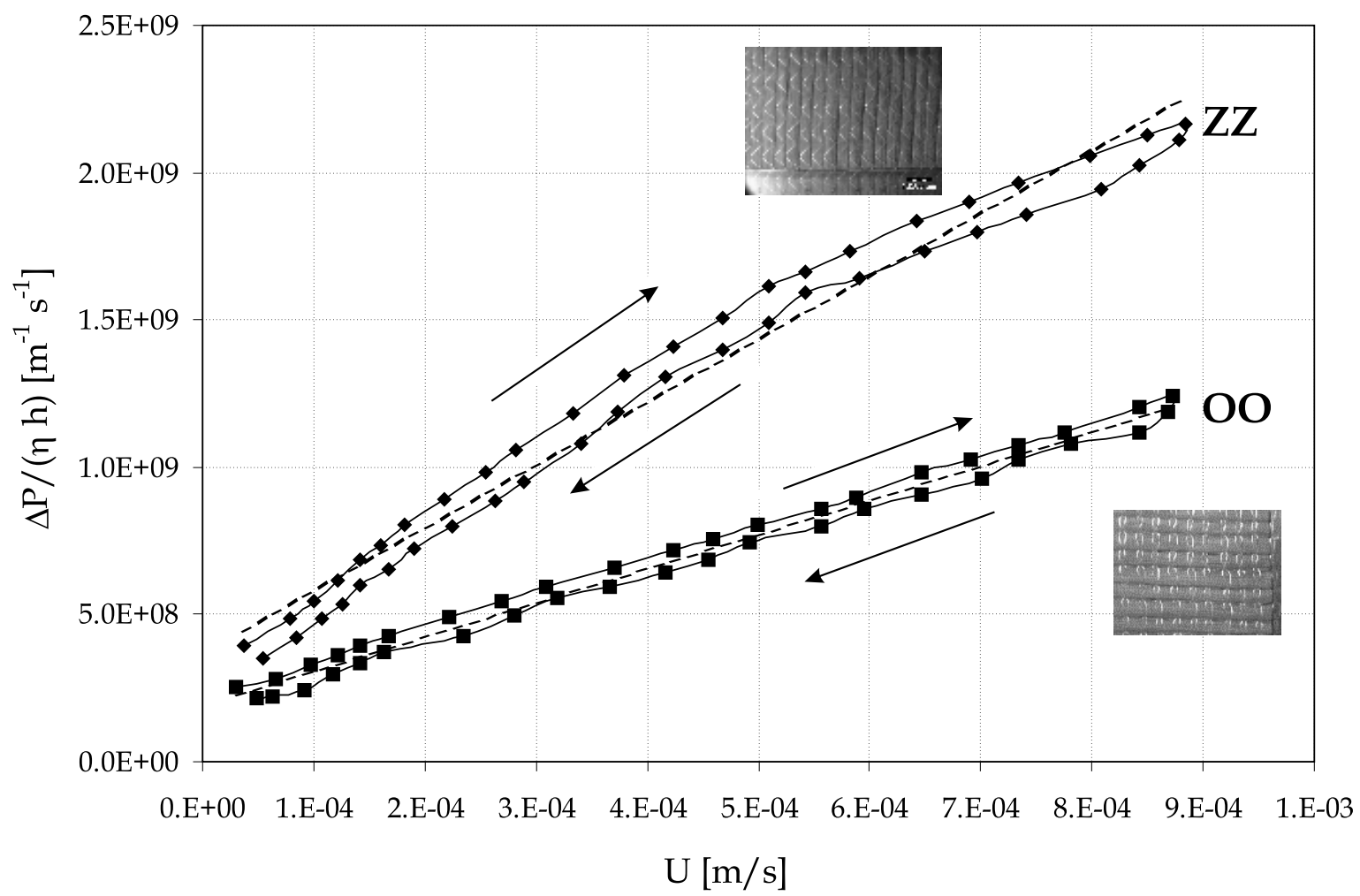

Figure 8: Resistance curves for Automotive $N C 2$ : inverse process and linear fit of the permeability. 


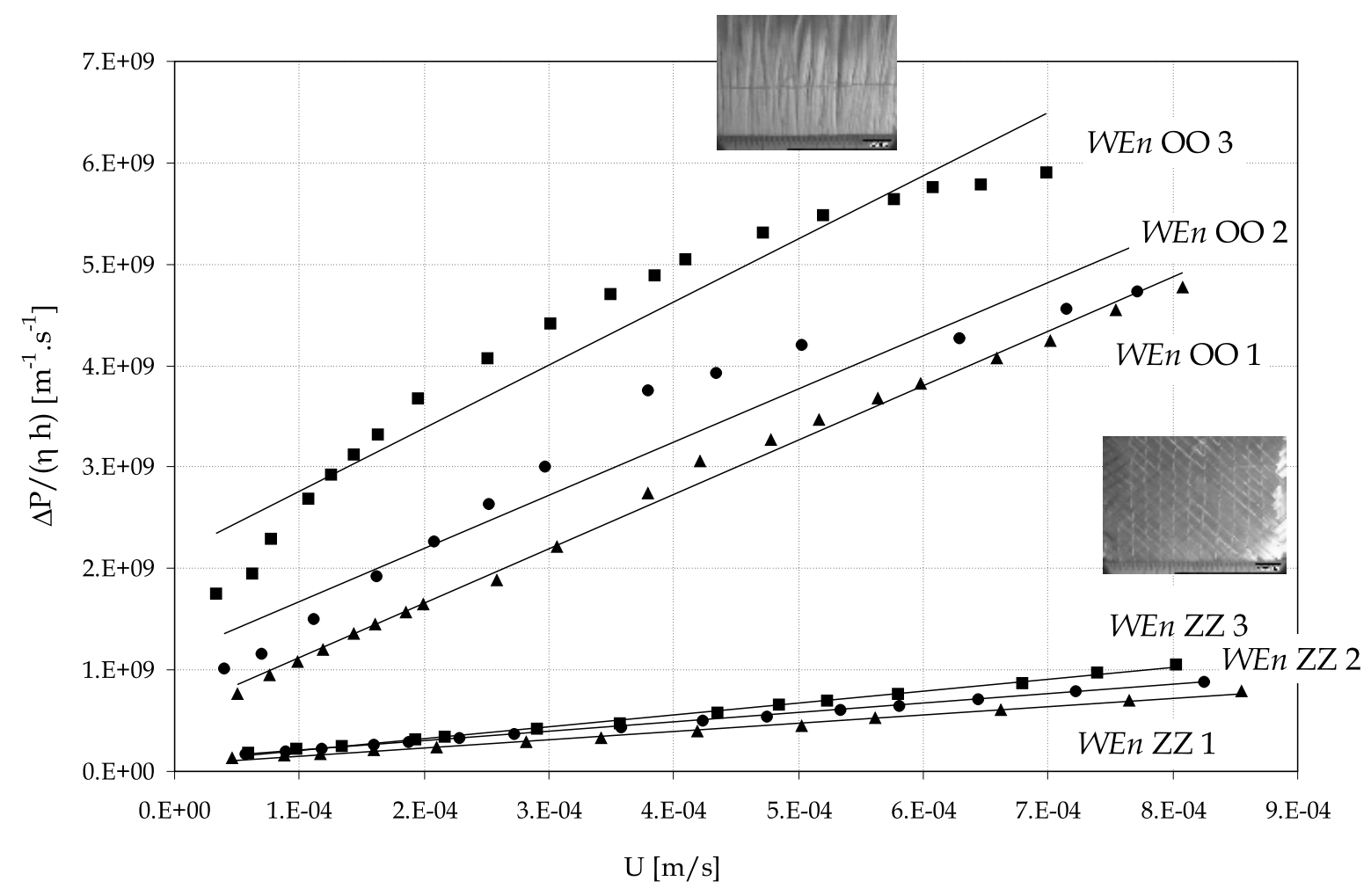

Figure 9: Resistance curves for Wind Energy $N C 2$. 


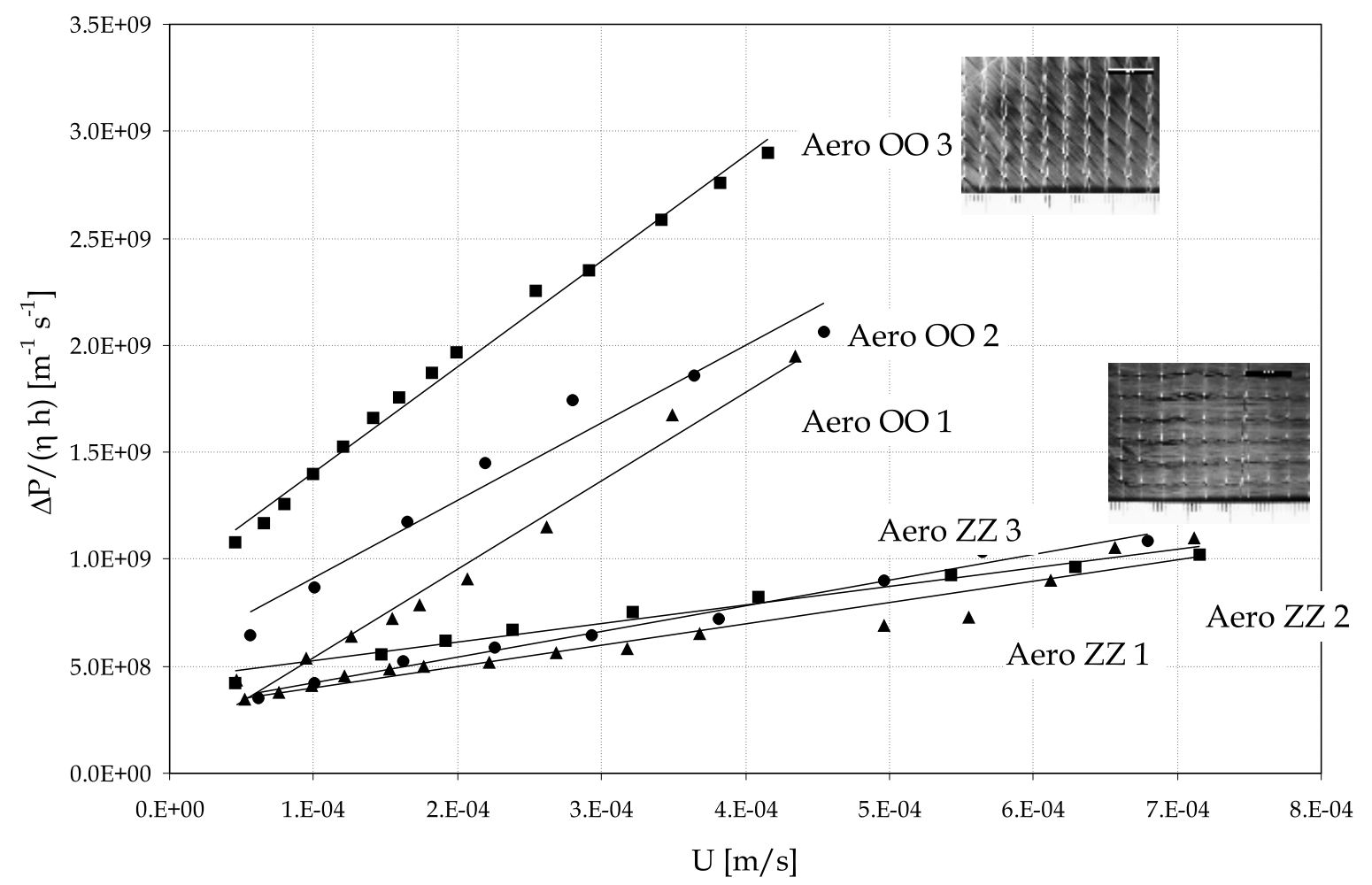

Figure 10: Resistance curves for Aeronautic NC2. 


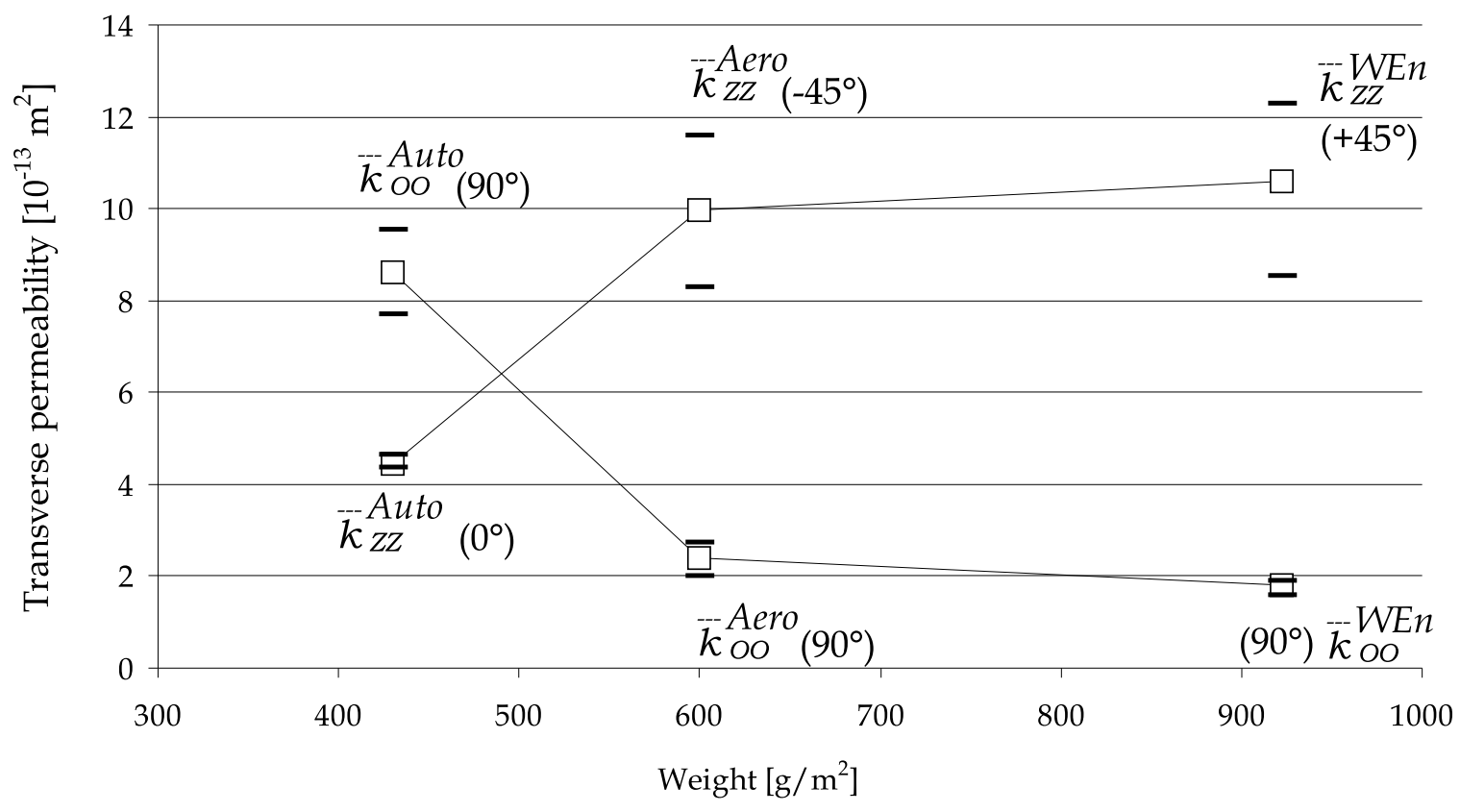

Figure 11: Transverse saturated permeabilities as functions of the $N C 2$ multiaxial weight (corresponding fibre orientation indicated between brackets).

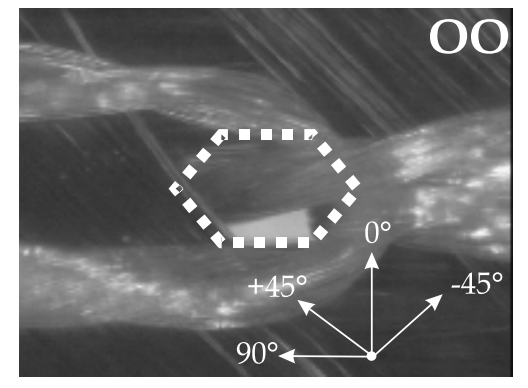

(a)

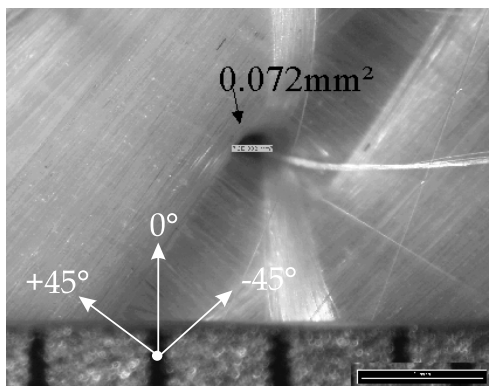

(b)

Figure 12: Example of stitching hole measurement surfaces in (a) transmission for Aeronautic $N C 2$ and (b) reflection for Wind Energy $N C 2$. 

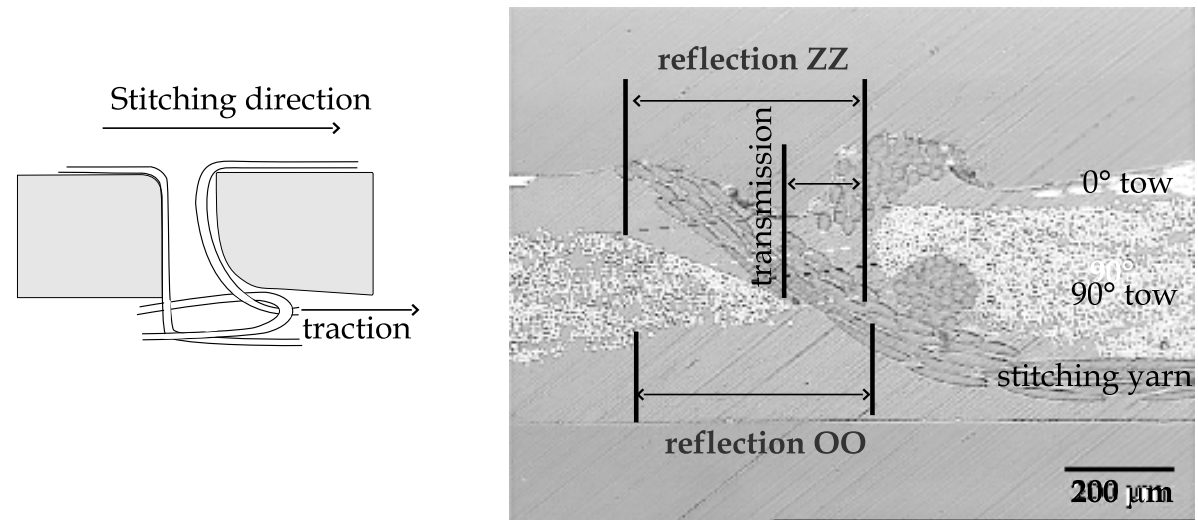

Figure 13: Conical shape of the stitching hole due to the stitching tension in biaxial Automotive $\mathrm{NC} 2$.

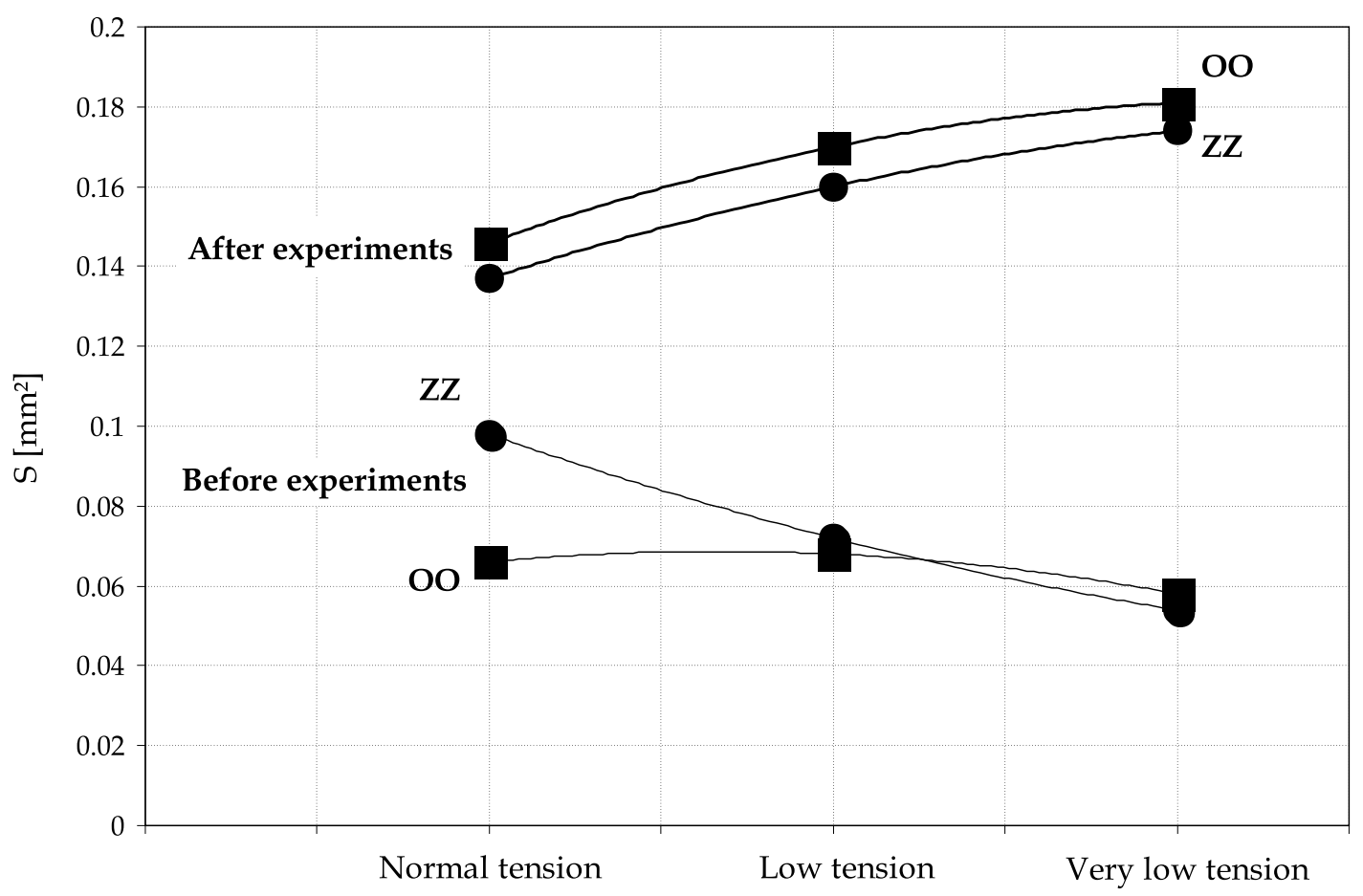

Figure 14: Stitching hole surface measurements in [+45/-45] NC2 BEFORE and AFTER permeability assessment experiments for varying stitching tension. 

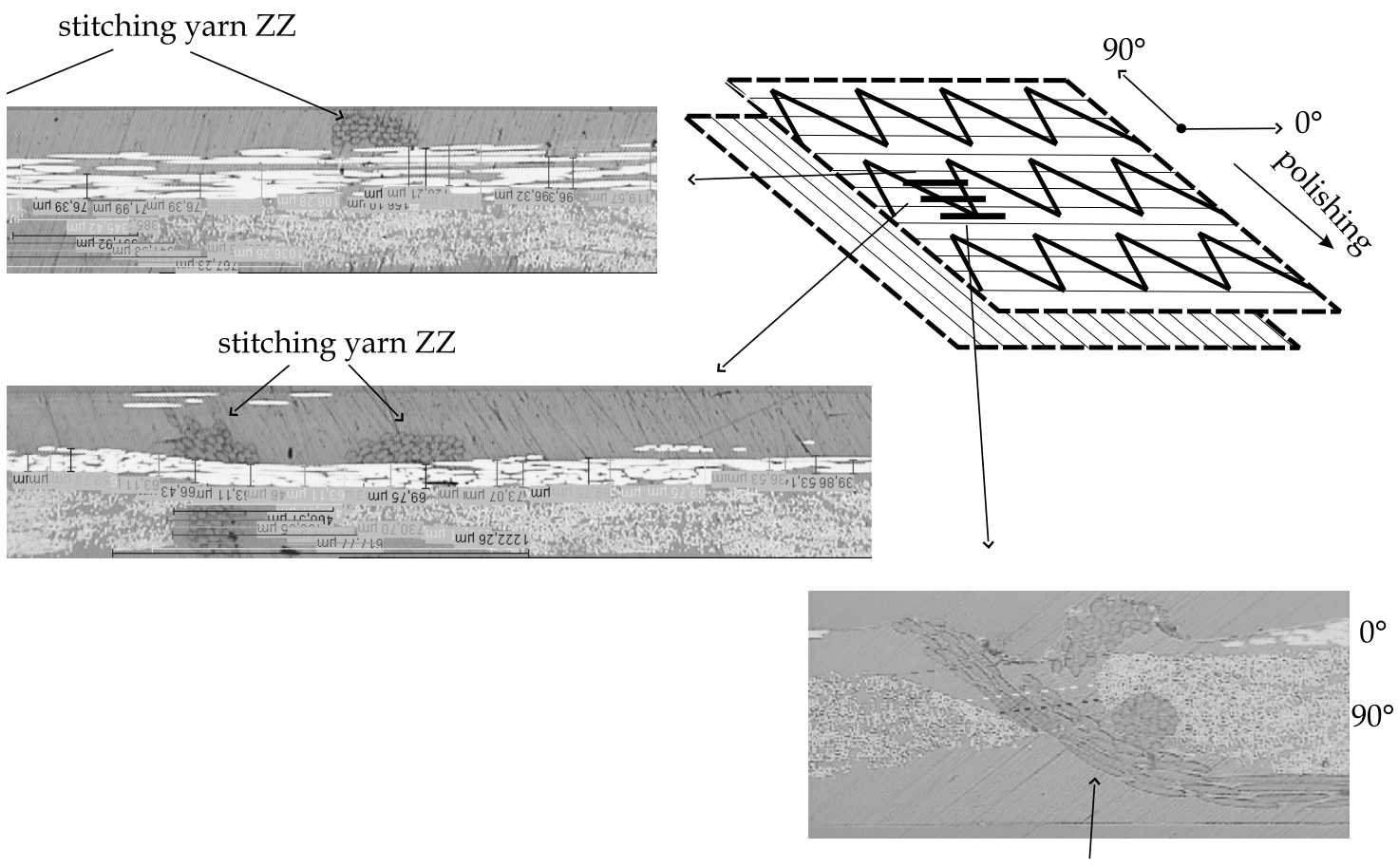

stitching yarn $\mathrm{OO}$

Figure 15: 3D reconstruction of a stitching hole in Automotive [0/90] NC2. 


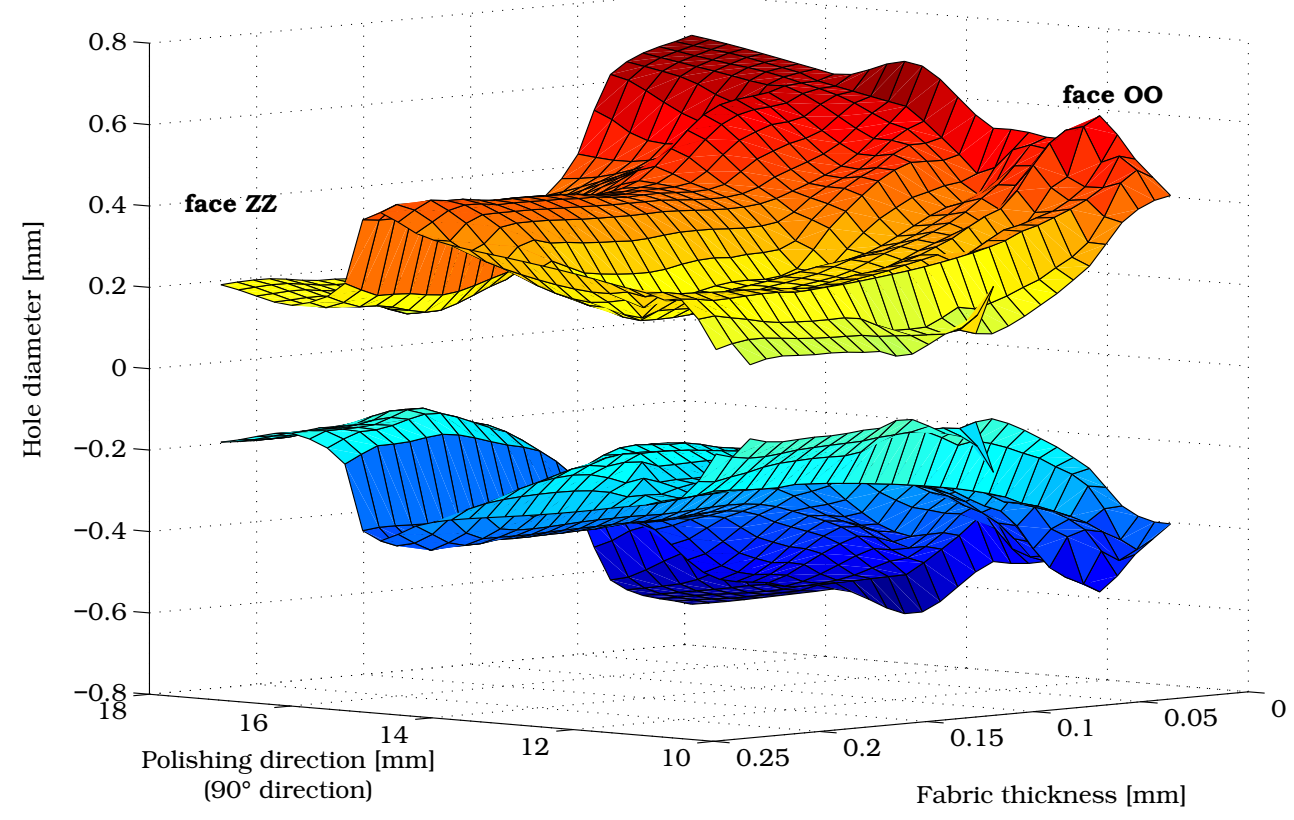

(a)

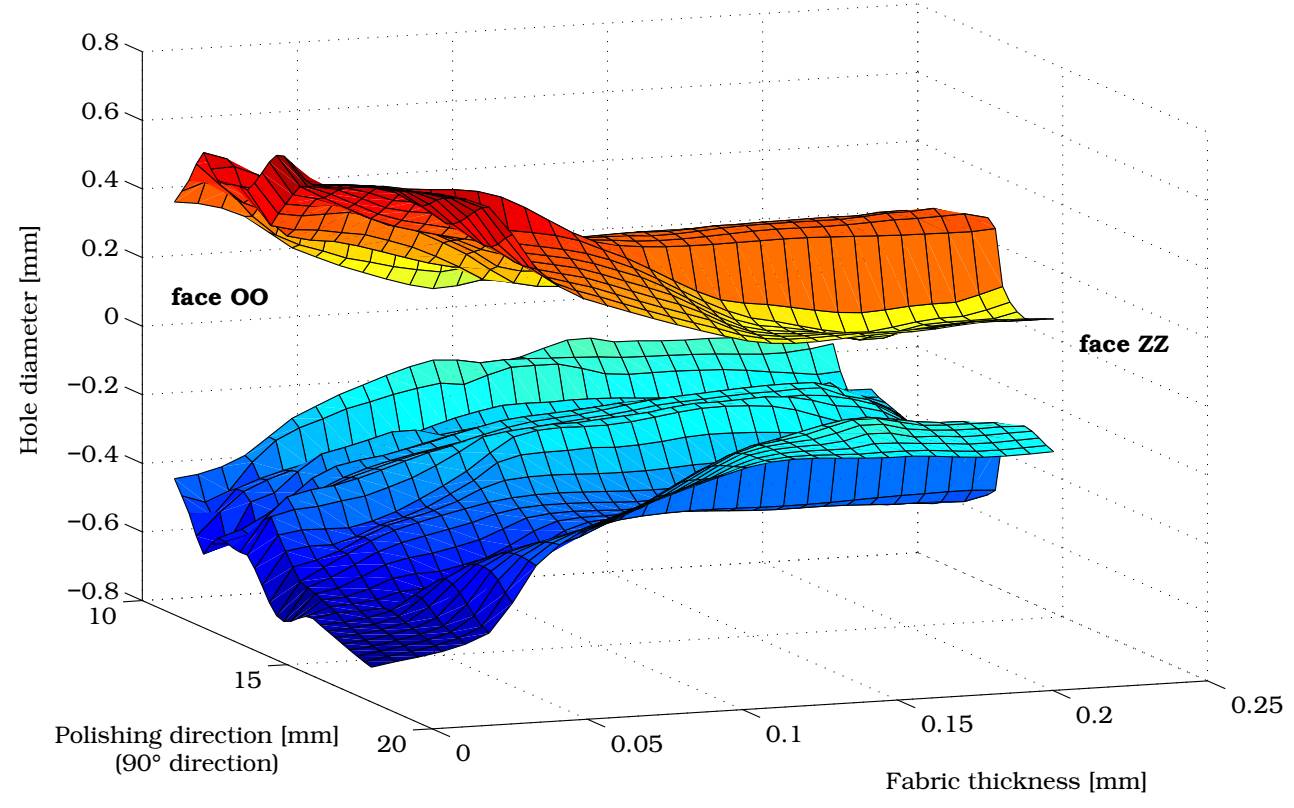

(b)

Figure 16: 3D reconstruction of a stitching hole in Automotive [0/90] NC2 : view (a) from the zig-zag face and (b) from the side. 